\title{
Stochastic Modelling of Tumor Growth within Organ during Chemotherapy Using Bivariate Birth, Death and Migration Processes
}

\author{
${ }^{1}$ Tirupathi Rao Padi, ${ }^{2}$ J. Jayabharathiraj, ${ }^{3}$ B.N. Naveen Kumar, \\ ${ }^{4}$ C. Lakshmi Usha, ${ }^{5}$ P. Rajasekhara Reddy \\ ${ }^{1,2}$ Dept. of Statistics, Pondicherry University Puducherry -605 014, India, \\ ${ }^{3,4,5}$ Dept. of Statistics, S.V. University Tirupathi - 517 502, Andhra Pradesh, India
}

\begin{abstract}
In this paper, a bivariate stochastic model for cancer growth within a specific organ during chemotherapy is developed using the birth, death and migration processes based on pathophysiology and genetic programs of cancerous cell. Joint probability functions and statistical properties of the model are derived with the formulated stochastic differential equations. Model behaviour was analysed with numerical data.
\end{abstract}

Keywords: Stochastic Modelling, Cancer Growth within Organ, Chemotherapy, Generalized Poisson Processes, Differential- difference equations

\section{Introduction}

Continuous proliferation with minimum rate of death in such cells will form a mass of accumulation of corrupted cells called tumour. The growth or loss processes of cells are depending on the nature of mutant cell and its stages of transformation. Invasion of cancer cells has a potential to generate new colonies at different sites of the body from the forming or hosting sites. Hence, the growth and spread of cancerous cells will have the random processes such as birth, death and migration. The dynamics of cancer cells spread is influenced by the drug presence and its absence during the treatment with chemotherapy. It is customary to assess the severity of the cancer of the patient through manual methods.

Approach of stochastic modelling for evaluating the health status of the patient will be more beneficial under the uncertain environment. There is much evidence in literature on quantitative approach of cancer growth studies. The colony size distribution of multiple metastatic tumor and their growth is modelled by Wata et al [1]. The cancer chemotherapy treatment with the metastasis is modelled mathematically by Pinho et al [2]. The growth of cancer during and after the chemotherapy is modelled for studying the equilibrium probability of tumor size by Srinivasa Rao et al [3,4]. Various stochastic multistage models were developed for dynamics of cells in the cancer tumor and its behaviour under the presence and absence of chemotherapy by Tirupathi Rao et al [5-7].

This study is focused on developing bi-variate stochastic model for the cancer cells growth in an organ under presence and absence of chemotherapy. The birth, death and migration of cells to the neighbouring parts of an organ have been considered in the model. The migration of cancer cells are happens through the process called metastasis. Initial position of cancer tumor is named as primary tumor and tumor in the neighbouring location due migration process is called secondary tumor. This model is constructed based on the biological and patho-physiological assumptions of cancer and completely randomized cell divisions. The following schematic diagram shall give more clear idea on cancer growth in presence and absence of chemotherapy.

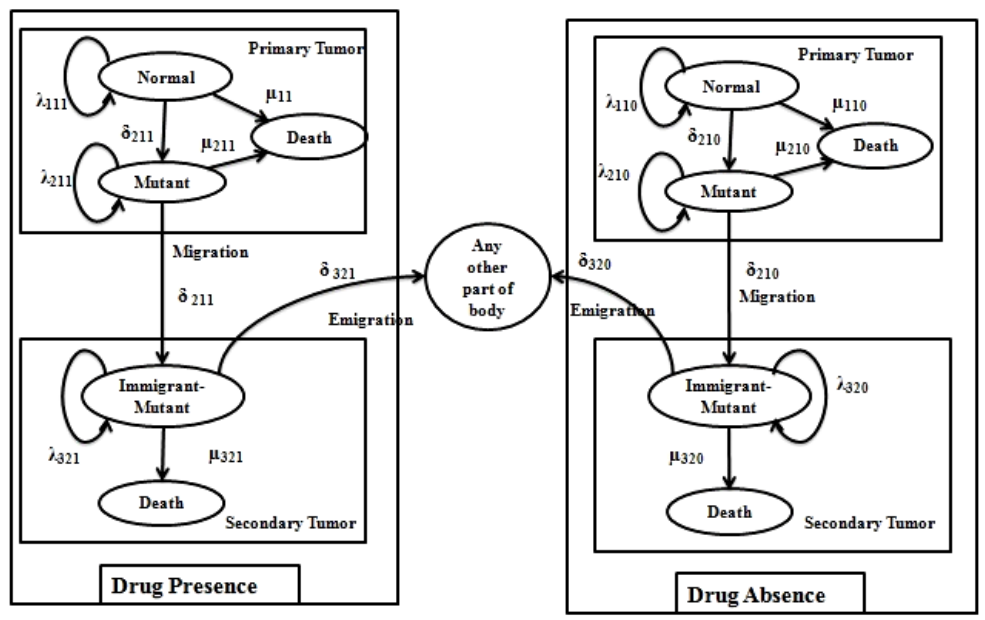

Figure -1: Schematic Diagram of cancer cells growth during chemotherapy. 


\section{Stochastic Model for growth of cancerous cell during chemotherapy}

The mechanisms involved in the cell divisions are purely stochastic in nature. Let the events occurred in non-overlapping interval of time are statistically independent. Let $\Delta t$ be an infinitesimal interval in the time.

Let $\lambda_{\mathrm{ijl}}$ be the growth rate of $\mathrm{i}^{\text {th }}$ stage cells in $\mathrm{j}^{\text {th }}$ stage tumor and $\mathrm{l}^{\text {th }}$ state of drug in chemotherapy; $\mu_{\mathrm{ijl}}$ be the loss rate of $\mathrm{i}^{\text {th }}$ stage cells in $\mathrm{j}^{\text {th }}$ stage tumor and $\mathrm{l}^{\text {th }}$ state of drug in chemotherapy;

$\delta_{\mathrm{ijl}}$ be the transformation rate of $\mathrm{i}^{\text {th }}$ stage cells to $(\mathrm{i}+1)^{\text {th }}$ stage in the $\mathrm{j}^{\text {th }}$ stage tumor to $(\mathrm{j}+1)^{\text {th }}$ stage of tumor and $1^{\text {th }}$ state of drug in chemotherapy.

Where,

$\mathrm{i}=1,2,3$ : Normal stage of cell, Mutant stage of cell, Migrant mutant stage of cell.

$\mathrm{j}=1,2$ : Primary stage of tumor, Secondary stage of tumor.

l=0, 1: Drug Absence, Drug Presence.

Let $\mathrm{a}_{\mathrm{k}}= \begin{cases}1 & \text { Drug Presence } \quad \text { for } \mathrm{k}=1,2,3,4,5,6,7,8,9 \\ 0 & \text { Drug Absence } \\ (0,1) & \text { Partial Presence of drug }\end{cases}$

Let $\{\mathrm{N}(\mathrm{t}), \mathrm{t} \geq 0\}$ be the process of normal cell division (growth/loss) and $\{\mathrm{M}(\mathrm{t}), \mathrm{t} \geq 0\}$ be the process of mutant cell division (growth/loss). Let $\{\mathrm{N}(\mathrm{t}), \mathrm{M}(\mathrm{t}), \mathrm{t} \geq 0\}$ be a joint bivariate stochastic processes of individual stochastic processes of $\{N(t), t \geq 0\}$ and $\{M(t), t \geq 0\}$. Such that $\operatorname{Pr}\{[N(t), M(t)]=[n, m]\}=P_{n, m}(t)$ and $\operatorname{Pr}\{\mathrm{N}(\mathrm{t})=\mathrm{n}\}=\mathrm{P}_{\mathrm{n}}(\mathrm{t}), \operatorname{Pr}\{\mathrm{M}(\mathrm{t})=\mathrm{m}\}=\mathrm{P}_{\mathrm{m}}(\mathrm{t})$.

Further,

$$
\begin{aligned}
& \operatorname{Pr}\{\mathrm{N}(\Delta \mathrm{t})=\mathrm{u} / \mathrm{N}(\mathrm{t})=\mathrm{n}\}=\mathrm{P}_{\mathrm{nu}} \text { for } \mathrm{u}=\mathrm{n}+1, \mathrm{n}-1, \mathrm{n}, \mathrm{n} \pm 2 \\
& \operatorname{Pr}\{\mathrm{M}(\Delta \mathrm{t})=\mathrm{v} / \mathrm{M}(\mathrm{t})=\mathrm{m}\}=\mathrm{P}_{\mathrm{mv}} \text { for } \mathrm{v}=\mathrm{m}+1, \mathrm{~m}-1, \mathrm{~m}, \mathrm{~m} \pm 2 \\
& \operatorname{Pr}\{\{[\mathrm{N}(\Delta \mathrm{t}), \mathrm{M}(\Delta \mathrm{t})]=(\mathrm{u}, \mathrm{v})\} /\{[\mathrm{N}(\mathrm{t}), \mathrm{M}(\mathrm{t})=(\mathrm{n}, \mathrm{m})]\}\}=\mathrm{P}_{\mathrm{nu}, \mathrm{mv}} \text { for } \mathrm{v}=\mathrm{m}+1, \mathrm{~m}-1, \mathrm{~m}, \mathrm{~m} \pm 2
\end{aligned}
$$

Let us now define postulates of the univariate process with respect to normal and mutant growth,

$$
\begin{array}{rlr}
\mathrm{P}_{\mathrm{n}, \mathrm{u}} & =\mathrm{P}\{\mathrm{N}(\Delta \mathrm{t})=\mathrm{u} / \mathrm{N}(\mathrm{t})=\mathrm{n}\} & \\
& =\mathrm{n}\left(\mathrm{a}_{1} \lambda_{111}+\left(1-\mathrm{a}_{1}\right) \lambda_{110}\right) \Delta \mathrm{t}+\mathrm{o}(\Delta \mathrm{t}) & ; \mathrm{u}=\mathrm{n}+1 \\
& =\mathrm{n}\left(\mathrm{a}_{5} \mu_{111}+\left(1-\mathrm{a}_{5}\right) \mu_{110}\right) \Delta \mathrm{t}+\mathrm{o}(\Delta \mathrm{t}) & ; \mathrm{u}=\mathrm{n}-1 \\
& =\mathrm{n}\left(\mathrm{a}_{2} \delta_{111}+\left(1-\mathrm{a}_{2}\right) \delta_{111}\right) \Delta \mathrm{t}+\mathrm{o}(\Delta \mathrm{t}) & \\
& =1-[\mathrm{u}=\mathrm{n}-1 \\
& \left.\mathrm{n}\left(\begin{array}{c}
\left(\mathrm{a}_{1} \lambda_{111}+\left(1-\mathrm{a}_{1}\right) \lambda_{110}\right) \\
+\left(\mathrm{a}_{5} \mu_{111}+\left(1-\mathrm{a}_{5}\right) \mu_{110}\right) \\
+\left(\mathrm{a}_{2} \delta_{111}+\left(1-\mathrm{a}_{2}\right) \delta_{111}\right)
\end{array}\right) \Delta \mathrm{t}+\mathrm{o}(\Delta \mathrm{t})\right] & ; \mathrm{u}=\mathrm{n} \\
& =\mathrm{o}(\Delta \mathrm{t})^{2} & \\
\end{array}
$$

For mutant growth processes,

$$
\begin{array}{rlrl}
\mathrm{P}_{\mathrm{m}, \mathrm{v}} & =\mathrm{P}\{\mathrm{M}(\Delta \mathrm{t})=\mathrm{v} / \mathrm{M}(\mathrm{t})=\mathrm{m}\} & \\
& =\mathrm{m}\left(\mathrm{a}_{3} \lambda_{211}+\left(1-\mathrm{a}_{3}\right) \lambda_{210}\right) \Delta \mathrm{t}+\mathrm{o}(\Delta \mathrm{t}) & & ; \mathrm{v}=\mathrm{m}+1 \\
& =\mathrm{m}\left(\mathrm{a}_{6} \mu_{211}+\left(1-\mathrm{a}_{6}\right) \mu_{210}\right) \Delta \mathrm{t}+\mathrm{o}(\Delta \mathrm{t}) & & ; \mathrm{v}=\mathrm{m}-1 \\
& =\mathrm{m}\left(\mathrm{a}_{7} \delta_{211}+\left(1-\mathrm{a}_{7}\right) \delta_{210}\right) \Delta \mathrm{t}+\mathrm{o}(\Delta \mathrm{t}) & & ; \mathrm{v}=\mathrm{m}-1 \\
& =\left(\mathrm{a}_{4} \lambda_{321}+\left(1-\mathrm{a}_{4}\right) \lambda_{320}\right) \Delta \mathrm{t}+\mathrm{o}(\Delta \mathrm{t}) & & ; \mathrm{v}=\mathrm{m}+1 \\
& =\left(\mathrm{a}_{8} \mu_{321}+\left(1-\mathrm{a}_{8}\right) \mu_{320}\right) \Delta \mathrm{t}+\mathrm{o}(\Delta \mathrm{t}) & ; \mathrm{v}=\mathrm{m}-1 \\
& =\left(\mathrm{a}_{9} \delta_{321}+\left(1-\mathrm{a}_{9}\right) \delta_{321}\right) \Delta \mathrm{t}+\mathrm{o}(\Delta \mathrm{t}) & \\
& =1-\left[\left\{\begin{array}{l}
\mathrm{m}\left(\left(\mathrm{a}_{3} \lambda_{211}+\left(1-\mathrm{a}_{3}\right) \lambda_{210}\right)+\left(\mathrm{a}_{6} \mu_{211}+\left(1-\mathrm{a}_{6}\right) \mu_{210}\right)\right. \\
\left.+\left(\mathrm{a}_{7} \delta_{211}+\left(1-\mathrm{a}_{7}\right) \delta_{210}\right)\right)+\left(\left(\mathrm{a}_{4} \lambda_{321}+\left(1-\mathrm{a}_{4}\right) \lambda_{320}\right)\right. \\
\left.+\left(\mathrm{a}_{8} \mu_{321}+\left(1-\mathrm{a}_{8}\right) \mu_{320}\right)+\left(\mathrm{a}_{9} \delta_{321}+\left(1-\mathrm{a}_{9}\right) \delta_{321}\right)\right)
\end{array}\right\} \Delta \mathrm{t}+\mathrm{o}(\Delta \mathrm{t})\right] ; \mathrm{v}=\mathrm{m} \\
& =o(\Delta \mathrm{t})^{2} & & ; \mathrm{v}=\mathrm{m} \pm 2
\end{array}
$$

Considering the joint stochastic processes, we have 


$$
\begin{aligned}
& \mathrm{P}_{\mathrm{nu}, \mathrm{mv}}=\mathrm{P}\{[(\mathrm{N}(\Delta \mathrm{t}), \mathrm{M}(\Delta \mathrm{t})]=(\mathrm{u}, \mathrm{v}) /[(\mathrm{N}(\mathrm{t}), \mathrm{M}(\mathrm{t})]=(\mathrm{n}, \mathrm{m})\} \\
& =n\left(a_{1} \lambda_{111}+\left(1-a_{1}\right) \lambda_{110}\right) \Delta t+o(\Delta t) \\
& =\mathrm{n}\left(\mathrm{a}_{5} \mu_{111}+\left(1-\mathrm{a}_{5}\right) \mu_{110}\right) \Delta \mathrm{t}+\mathrm{o}(\Delta \mathrm{t}) \\
& =\mathrm{n}\left(\mathrm{a}_{2} \delta_{111}+\left(1-\mathrm{a}_{2}\right) \delta_{111}\right) \Delta \mathrm{t}+\mathrm{o}(\Delta \mathrm{t}) \\
& =\mathrm{m}\left(\mathrm{a}_{3} \lambda_{211}+\left(1-\mathrm{a}_{3}\right) \lambda_{210}\right) \Delta \mathrm{t}+\mathrm{o}(\Delta \mathrm{t}) \\
& =\mathrm{m}\left(\mathrm{a}_{6} \mu_{211}+\left(1-\mathrm{a}_{6}\right) \mu_{210}\right) \Delta \mathrm{t}+\mathrm{o}(\Delta \mathrm{t}) \\
& =\mathrm{m}\left(\mathrm{a}_{7} \delta_{211}+\left(1-\mathrm{a}_{7}\right) \delta_{210}\right) \Delta \mathrm{t}+\mathrm{o}(\Delta \mathrm{t}) \\
& =\left(\mathrm{a}_{4} \lambda_{321}+\left(1-\mathrm{a}_{4}\right) \lambda_{320}\right) \Delta \mathrm{t}+\mathrm{o}(\Delta \mathrm{t}) \\
& =\left(\mathrm{a}_{8} \mu_{321}+\left(1-\mathrm{a}_{8}\right) \mu_{320}\right) \Delta \mathrm{t}+\mathrm{o}(\Delta \mathrm{t}) \\
& =\left(a_{9} \delta_{321}+\left(1-a_{9}\right) \delta_{320}\right) \Delta t+o(\Delta t) \\
& ; \mathrm{u}=\mathrm{n}+1, \mathrm{v}=\mathrm{m} \\
& ; \mathrm{u}=\mathrm{n}-1, \mathrm{v}=\mathrm{m} \\
& ; \mathrm{u}=\mathrm{n}-1, \mathrm{v}=\mathrm{m} \\
& ; \mathrm{u}=\mathrm{n}, \mathrm{v}=\mathrm{m}+1 \\
& ; \mathrm{u}=\mathrm{n}, \mathrm{v}=\mathrm{m}-1 \\
& ; \mathrm{u}=\mathrm{n}, \mathrm{v}=\mathrm{m}-1 \\
& ; \mathrm{u}=\mathrm{n}, \mathrm{v}=\mathrm{m}+1 \\
& ; \mathrm{u}=\mathrm{n}, \mathrm{v}=\mathrm{m}-1 \\
& =1-\left[\begin{array}{l}
\mathrm{n}\left(\left(\mathrm{a}_{1} \lambda_{111}+\left(1-\mathrm{a}_{1}\right) \lambda_{110}\right)+\left(\mathrm{a}_{5} \mu_{111}+\left(1-\mathrm{a}_{5}\right) \mu_{110}\right)\right. \\
\left.+\left(\mathrm{a}_{2} \delta_{111}+\left(1-\mathrm{a}_{2}\right) \delta_{111}\right)\right)+\mathrm{m}\left(\left(\mathrm{a}_{3} \lambda_{211}+\left(1-\mathrm{a}_{3}\right) \lambda_{210}\right)\right. \\
\left.+\left(\mathrm{a}_{6} \mu_{211}+\left(1-\mathrm{a}_{6}\right) \mu_{210}\right)+\left(\mathrm{a}_{7} \delta_{211}+\left(1-\mathrm{a}_{7}\right) \delta_{210}\right)\right) \\
+\left(\left(\mathrm{a}_{4} \lambda_{321}+\left(1-\mathrm{a}_{4}\right) \lambda_{320}\right)+\left(\mathrm{a}_{8} \mu_{321}+\left(1-\mathrm{a}_{8}\right) \mu_{320}\right)\right. \\
\left.+\left(\mathrm{a}_{9} \delta_{321}+\left(1-\mathrm{a}_{9}\right) \delta_{320}\right)\right)
\end{array}\right\} \\
& ; \mathrm{u}=\mathrm{n}, \mathrm{v}=\mathrm{m}-1 \\
& =\mathrm{o}(\Delta \mathrm{t})^{2} \\
& ; \mathrm{u}=\mathrm{n}, \mathrm{v}=\mathrm{m} \\
& ; \mathrm{u}=\mathrm{n} \pm 2, \mathrm{v}=\mathrm{m} \pm 2
\end{aligned}
$$

Let $\mathrm{P}_{n, m}(t+\Delta t)$ be the probability that happening of an event of one event in an infinitesimal interval $\Delta t$, there exists ' $\mathrm{n}$ ' normal and ' $\mathrm{m}$ ' mutant cells in the organ upto time ' $\mathrm{t}$ '. Then the differential - difference equations of the model are:

$$
\begin{aligned}
& \mathrm{P}_{\mathrm{n}, \mathrm{m}}^{\prime}(\mathrm{t})=-\left\{\mathrm{n}\left(\left(\mathrm{a}_{1} \lambda_{111}+\left(1-\mathrm{a}_{1}\right) \lambda_{110}\right)+\left(\mathrm{a}_{2} \delta_{111}+\left(1-\mathrm{a}_{2}\right) \delta_{110}\right)+\left(\mathrm{a}_{5} \mu_{111}+\left(1-\mathrm{a}_{5}\right) \mu_{110}\right)\right)\right. \\
& +\mathrm{m}\left(\left(\mathrm{a}_{3} \lambda_{211}+\left(1-\mathrm{a}_{3}\right) \lambda_{210}\right)+\left(\mathrm{a}_{6} \mu_{211}+\left(1-\mathrm{a}_{6}\right) \mu_{210}\right)+\left(\mathrm{a}_{7} \delta_{211}+\left(1-\mathrm{a}_{7}\right) \delta_{210}\right)\right) \\
& \left.+\left(\left(a_{4} \lambda_{41}+\left(1-a_{4}\right) \lambda_{40}\right)+\left(a_{8} \mu_{41}+\left(1-a_{8}\right) \mu_{40}\right)+\left(a_{9} \mu_{51}+\left(1-a_{9}\right) \mu_{50}\right)\right)\right\} P_{n, m}(t) \\
& +\mathrm{P}_{\mathrm{n}+1, \mathrm{~m}}(\mathrm{t})\left[(\mathrm{n}-1)\left(\mathrm{a}_{1} \lambda_{111}+\left(1-\mathrm{a}_{1}\right) \lambda_{110}\right)\right]+\mathrm{P}_{\mathrm{n}+1, \mathrm{~m}-1}(\mathrm{t})\left[(\mathrm{n}+1)\left(\mathrm{a}_{2} \delta_{111}+\left(1-\mathrm{a}_{2}\right) \delta_{110}\right)\right] \\
& +\mathrm{P}_{\mathrm{n}, \mathrm{m}-1}(\mathrm{t})\left[(\mathrm{m}-1)\left(\mathrm{a}_{3} \lambda_{211}+\left(1-\mathrm{a}_{3}\right) \lambda_{210}\right)\right]+\mathrm{P}_{\mathrm{n}+1, \mathrm{~m}}(\mathrm{t})\left[(\mathrm{n}+1)\left(\mathrm{a}_{5} \mu_{111}+\left(1-\mathrm{a}_{5}\right) \mu_{110}\right)\right] \\
& +\mathrm{P}_{\mathrm{n}, \mathrm{m}+1}(\mathrm{t})\left[(\mathrm{m}+1)\left(\mathrm{a}_{6} \mu_{211}+\left(1-\mathrm{a}_{6}\right) \mu_{210}\right)\right]+\mathrm{P}_{\mathrm{n}, \mathrm{m}+1}(\mathrm{t})\left[(\mathrm{m}+1)\left(\mathrm{a}_{7} \delta_{211}+\left(1-\mathrm{a}_{7}\right) \delta_{210}\right)\right] \\
& +\mathrm{P}_{\mathrm{n}, \mathrm{m}-1}(\mathrm{t})\left[\left(\mathrm{a}_{4} \lambda_{321}+\left(1-\mathrm{a}_{4}\right) \lambda_{320}\right)\right]+\mathrm{P}_{\mathrm{n}, \mathrm{m}+1}(\mathrm{t})\left[\left(\mathrm{a}_{8} \mu_{321}+\left(1-\mathrm{a}_{8}\right) \mu_{320}\right)\right] \\
& +\mathrm{P}_{\mathrm{n}, \mathrm{m}+1}(\mathrm{t})\left[\left(\mathrm{a}_{9} \delta_{321}+\left(1-\mathrm{a}_{9}\right) \delta_{320}\right)\right] \quad \text { for } \mathrm{n}, \mathrm{m} \geq 1 \\
& \mathrm{P}_{0,1}^{\prime}(\mathrm{t})=-\left[\left(\mathrm{a}_{3} \lambda_{211}+\left(1-\mathrm{a}_{3}\right) \lambda_{210}\right)+\left(\mathrm{a}_{4} \lambda_{321}+\left(1-\mathrm{a}_{4}\right) \lambda_{320}\right)+\left(\mathrm{a}_{6} \mu_{211}+\left(1-\mathrm{a}_{6}\right) \mu_{210}\right)\right. \\
& \left.+\left(a_{8} \mu_{321}+\left(1-a_{8}\right) \mu_{320}\right)+\left(a_{9} \delta_{321}+\left(1-a_{9}\right) \delta_{320}\right)\right] P_{0,1}(t)+\left(a_{5} \mu_{111}+\left(1-a_{5}\right) \mu_{110}\right) P_{1,1}(t) \\
& +\left(\mathrm{a}_{2} \delta_{111}+\left(1-\mathrm{a}_{2}\right) \delta_{110}\right) \mathrm{P}_{1,0}(\mathrm{t})+\left\{2\left(\left(\mathrm{a}_{6} \mu_{211}+\left(1-\mathrm{a}_{6}\right) \mu_{210}\right)+\left(\mathrm{a}_{7} \delta_{211}+\left(1-\mathrm{a}_{7}\right) \delta_{210}\right)\right)\right. \\
& \left.+\left(\mathrm{a}_{8} \mu_{321}+\left(1-\mathrm{a}_{8}\right) \mu_{320}\right)+\left(\mathrm{a}_{9} \delta_{321}+\left(1-\mathrm{a}_{9}\right) \delta_{320}\right)\right\} \mathrm{P}_{0,2}(\mathrm{t})+\left(\mathrm{a}_{4} \lambda_{321}+\left(1-\mathrm{a}_{4}\right) \lambda_{320}\right) \mathrm{P}_{0,0}(\mathrm{t}) \\
& \mathrm{P}_{1,0}^{\prime}(\mathrm{t})=\mathrm{P}_{1,0}(\mathrm{t})\left\{-\left(\left(\mathrm{a}_{1} \lambda_{111}+\left(1-\mathrm{a}_{1}\right) \lambda_{110}\right)+\left(\mathrm{a}_{2} \delta_{111}+\left(1-\mathrm{a}_{2}\right) \delta_{110}\right)+\left(\mathrm{a}_{4} \lambda_{321}+\left(1-\mathrm{a}_{4}\right) \lambda_{320}\right)\right.\right. \\
& +\left(a_{5} \mu_{111}+\left(1-a_{5}\right) \mu_{110}\right)+\left(a_{7} \delta_{211}+\left(1-a_{7}\right) \delta_{210}\right)+\left(a_{8} \mu_{321}+\left(1-a_{8}\right) \mu_{320}\right)+\left(a_{9} \delta_{321}\right. \\
& \left.\left.\left.+\left(1-\mathrm{a}_{9}\right) \delta_{320}\right)\right)\right\}+2\left(\mathrm{a}_{5} \mu_{111}+\left(1-\mathrm{a}_{5}\right) \mu_{110}\right) \mathrm{P}_{2,0}(\mathrm{t})+\mathrm{P}_{1,1}(\mathrm{t})\left\{\left(\left(\mathrm{a}_{5} \mu_{111}+\left(1-\mathrm{a}_{5}\right) \mu_{110}\right)\right.\right. \\
& \left.\left.+\left(a_{7} \delta_{211}+\left(1-a_{7}\right) \delta_{210}\right)+\left(a_{8} \mu_{321}+\left(1-a_{8}\right) \mu_{320}\right)+\left(a_{9} \delta_{321}+\left(1-a_{9}\right) \delta_{320}\right)\right)\right\} \\
& \mathrm{P}_{0.0}^{\prime}(\mathrm{t})=\left\{-\left[\left(\mathrm{a}_{4} \lambda_{321}+\left(1-\mathrm{a}_{4}\right) \lambda_{320}\right)+\left(\mathrm{a}_{8} \mu_{321}+\left(1-\mathrm{a}_{8}\right) \mu_{320}\right)+\left(\mathrm{a}_{9} \delta_{321}+\left(1-\mathrm{a}_{9}\right) \delta_{320}\right)\right]\right\} \mathrm{P}_{0,0}(\mathrm{t}) \\
& +\left(a_{5} \mu_{111}+\left(1-a_{5}\right) \mu_{110}\right) P_{1,0}(t)+\left(\left(a_{5} \mu_{111}+\left(1-a_{5}\right) \mu_{110}\right)+\left(a_{7} \delta_{211}+\left(1-a_{7}\right) \delta_{210}\right)\right. \\
& \left.+\left(a_{8} \mu_{321}+\left(1-a_{8}\right) \mu_{320}\right)+\left(a_{9} \delta_{321}+\left(1-a_{9}\right) \delta_{320}\right)\right) P_{0,1}(t)
\end{aligned}
$$

With the initial condition 
$\mathrm{P}_{\mathrm{N}_{0}, \mathrm{M}_{0}}(\mathrm{t})=1, \mathrm{P}_{\mathrm{i}, \mathrm{j}}(0)=0 \quad \forall \mathrm{i} \neq \mathrm{N}_{0} ; \mathrm{j} \neq \mathrm{M}_{0}$

\section{Generating Functions and Statistical Measures}

Let $P(x, y ; t)$ be the probability generating function of $P_{n, m}(t)$.

Where, $\left.P(x, y ; t)=\sum_{m=0}^{\infty} \sum_{n=0}^{\infty} x^{n} y^{m} P_{n, m}(t)\right) ;|x|<1,|y|<1$. Multiplying the above differential-difference equations (2.1) to (2.4) with $x^{n} y^{m}$ and summing over $\mathrm{n}$, m, we get

$$
\begin{aligned}
\frac{d}{d x} P(x, y ; t)= & -\left(\left(a_{1} \lambda_{111}+\left(1-a_{1}\right) \lambda_{110}\right)+\left(a_{2} \delta_{111}+\left(1-a_{2}\right) \delta_{110}\right)+\left(a_{5} \mu_{111}+\left(1-a_{5}\right) \mu_{110}\right)\right) x \sum_{m=0}^{\infty} \sum_{n=0}^{\infty} n x^{n-1} y^{m} P_{n, m}(t) \\
& -\left(\left(a_{3} \lambda_{211}+\left(1-a_{3}\right) \lambda_{210}\right)+\left(a_{6} \mu_{211}+\left(1-a_{6}\right) \mu_{210}\right)+\left(a_{7} \delta_{211}+\left(1-a_{7}\right) \delta_{210}\right)\right) y \sum_{m=0}^{\infty} \sum_{n=0}^{\infty} m x^{n} y^{m-1} P_{n, m}(t) \\
& -\left(\left(a_{4} \lambda_{321}+\left(1-a_{4}\right) \lambda_{320}\right)+\left(a_{8} \mu_{321}+\left(1-a_{8}\right) \mu_{320}\right)+\left(a_{9} \delta_{321}+\left(1-a_{9}\right) \delta_{320}\right)\right) \sum_{m=0}^{\infty} \sum_{\mathrm{n}=0}^{\infty} x^{n} y^{m} P_{n, m}(t) \\
& +\left(a_{1} \lambda_{111}+\left(1-a_{1}\right) \lambda_{110}\right) x^{2} \sum_{m=0}^{\infty} \sum_{n=0}^{\infty}(n-1) x^{n-2} y^{m} P_{n+1, m}(t)+\left(a_{2} \delta_{111}+\left(1-a_{2}\right) \delta_{110}\right) y \sum_{m=0}^{\infty} \sum_{n=0}^{\infty}(n+1) x^{n} y^{m-1} \\
& P_{n+1, m-1}(t)+\left(a_{3} \lambda_{211}+\left(1-a_{3}\right) \lambda_{210}\right) y^{2} \sum_{m=0}^{\infty} \sum_{n=0}^{\infty}(m-1) x^{n} y^{m-2} P_{n, m-1}(t)+\left(a_{5} \mu_{111}+\left(1-a_{5}\right) \mu_{110}\right) \\
& \sum_{m=0}^{\infty} \sum_{n=0}^{\infty}(n+1) x^{n} y^{m} P_{n+1, m}(t)+\left(\left(a_{6} \mu_{211}+\left(1-a_{6}\right) \mu_{210}\right)+\left(a_{7} \delta_{211}+\left(1-a_{7}\right) \delta_{210}\right)\right) \sum_{m=0}^{\infty} \sum_{n=0}^{\infty}(m+1) x^{n} y^{m} \\
& P_{n+1, m-1}(t)+\left(a_{3} \lambda_{211}+\left(1-a_{3}\right) \lambda_{210}\right) y^{2} \sum_{m=0}^{\infty} \sum_{n=0}^{\infty}(m-1) x^{n} y^{m-2} P_{n, m-1}(t)+\left(a_{5} \mu_{111}+\left(1-a_{5}\right) \mu_{110}\right) \\
& \sum_{m=0}^{\infty} \sum_{n=0}^{\infty}(n+1) x^{n} y^{m} P_{n+1, m}(t)+\left(\left(a_{6} \mu_{211}+\left(1-a_{6}\right) \mu_{210}\right)+\left(a_{7} \delta_{211}+\left(1-a_{7}\right) \delta_{210}\right)\right) \sum_{m=0}^{\infty} \sum_{n=0}^{\infty}(m+1) x^{n} y^{m}
\end{aligned}
$$

On simplification, we obtain the differential equation of the form as follows,

$$
\begin{aligned}
\frac{\partial}{\partial \mathrm{t}} \mathrm{P}(\mathrm{x}, \mathrm{y} ; \mathrm{t})=\{ & -\left(\left(\mathrm{a}_{1} \lambda_{111}+\left(1-\mathrm{a}_{1}\right) \lambda_{110}\right)+\left(\mathrm{a}_{2} \delta_{111}+\left(1-\mathrm{a}_{2}\right) \delta_{110}\right)+\left(\mathrm{a}_{5} \mu_{111}+\left(1-\mathrm{a}_{5}\right) \mu_{110}\right)\right) \mathrm{x}+\left(\mathrm{a}_{5} \mu_{111}\right. \\
& \left.\left.+\left(1-\mathrm{a}_{5}\right) \mu_{110}\right)+\left(\mathrm{a}_{1} \lambda_{111}+\left(1-\mathrm{a}_{1}\right) \lambda_{110}\right) \mathrm{x}^{2}+\left(\mathrm{a}_{2} \delta_{111}+\left(1-\mathrm{a}_{2}\right) \delta_{110}\right) \mathrm{y}\right\} \frac{\partial}{\partial \mathrm{x}} \mathrm{P}(\mathrm{x}, \mathrm{y} ; \mathrm{t}) \\
& +\left\{-\left(\left(\mathrm{a}_{3} \lambda_{211}+\left(1-\mathrm{a}_{3}\right) \lambda_{210}\right)+\left(\mathrm{a}_{6} \mu_{211}+\left(1-\mathrm{a}_{6}\right) \mu_{210}\right)+\left(\mathrm{a}_{7} \delta_{211}+\left(1-\mathrm{a}_{7}\right) \delta_{210}\right)\right) \mathrm{y}\right. \\
& \left.+\left(\mathrm{a}_{3} \lambda_{211}+\left(1-\mathrm{a}_{3}\right) \lambda_{210}\right) \mathrm{y}^{2}+\left(\left(\mathrm{a}_{6} \mu_{211}+\left(1-\mathrm{a}_{6}\right) \mu_{210}\right)+\left(\mathrm{a}_{7} \delta_{211}+\left(1-\mathrm{a}_{7}\right) \delta_{210}\right)\right)\right\} \\
& \frac{\partial}{\partial \mathrm{y}} \mathrm{P}(\mathrm{x}, \mathrm{y} ; \mathrm{t})+\left\{-\left(\left(\mathrm{a}_{4} \lambda_{321}+\left(1-\mathrm{a}_{4}\right) \lambda_{320}\right)+\left(\mathrm{a}_{8} \mu_{321}+\left(1-\mathrm{a}_{8}\right) \mu_{320}\right)\right.\right. \\
& \left.+\left(\mathrm{a}_{9} \delta_{321}+\left(1-\mathrm{a}_{9}\right) \delta_{320}\right)\right)+\frac{\left[\left(\mathrm{a}_{8} \mu_{321}+\left(1-\mathrm{a}_{8}\right) \mu_{320}\right)+\left(\mathrm{a}_{9} \delta_{321}+\left(1-\mathrm{a}_{9}\right) \delta_{320}\right)\right]}{\mathrm{y}} \\
& \left.+\left(\mathrm{a}_{4} \lambda_{321}+\left(1-\mathrm{a}_{4}\right) \lambda_{320}\right) \mathrm{y}\right\} \mathrm{P}(\mathrm{x}, \mathrm{y} ; \mathrm{t})
\end{aligned}
$$

We can obtain the characteristics of the model using joint cumulant generating function of $P_{n, m}(t)$. Taking $\mathrm{x}=\mathrm{e}^{\mathrm{u}}, \mathrm{y}=\mathrm{e}^{\mathrm{v}}$ and denoting $\mathrm{k}(\mathrm{u}, \mathrm{v} ; \mathrm{t})$ as the joint cumulant generating function of $\mathrm{P}_{\mathrm{n}, \mathrm{m}}(\mathrm{t})$, we get the following expression

$$
\begin{aligned}
\frac{\partial}{\partial \mathrm{t}} \mathrm{k}(\mathrm{u}, \mathrm{v} ; \mathrm{t})= & \left\{-\left(\left(\mathrm{a}_{1} \lambda_{111}+\left(1-\mathrm{a}_{1}\right) \lambda_{110}\right)+\left(\mathrm{a}_{2} \delta_{111}+\left(1-\mathrm{a}_{2}\right) \delta_{110}\right)+\left(\mathrm{a}_{5} \mu_{111}+\left(1-\mathrm{a}_{5}\right) \mu_{110}\right)\right)+\left(\mathrm{a}_{5} \mu_{111}\right.\right. \\
& \left.\left.+\left(1-\mathrm{a}_{5}\right) \mu_{110}\right)+\left(\mathrm{a}_{1} \lambda_{111}+\left(1-\mathrm{a}_{1}\right) \lambda_{110}\right) \mathrm{e}^{\mathrm{u}}+\left(\mathrm{a}_{2} \delta_{111}+\left(1-\mathrm{a}_{2}\right) \delta_{110}\right) \mathrm{e}^{\mathrm{v}-\mathrm{u}}\right\} \frac{\partial}{\partial \mathrm{u}} \mathrm{k}(\mathrm{u}, \mathrm{v} ; \mathrm{t}) \\
& +\left\{-\left(\left(\mathrm{a}_{3} \lambda_{211}+\left(1-\mathrm{a}_{3}\right) \lambda_{210}\right)+\left(\mathrm{a}_{6} \mu_{211}+\left(1-\mathrm{a}_{6}\right) \mu_{210}\right)+\left(\mathrm{a}_{7} \delta_{211}+\left(1-\mathrm{a}_{7}\right) \delta_{210}\right)\right)\right.
\end{aligned}
$$




$$
\begin{aligned}
& \left.+\left(\mathrm{a}_{3} \lambda_{211}+\left(1-\mathrm{a}_{3}\right) \lambda_{210}\right) \mathrm{e}^{\mathrm{v}}+\left(\left(\mathrm{a}_{6} \mu_{211}+\left(1-\mathrm{a}_{6}\right) \mu_{210}\right)+\left(\mathrm{a}_{7} \delta_{211}+\left(1-\mathrm{a}_{7}\right) \delta_{210}\right)\right) \mathrm{e}^{-\mathrm{v}}\right\} \\
& \frac{\partial}{\partial \mathrm{v}} \mathrm{k}(\mathrm{u}, \mathrm{v} ; \mathrm{t})+\left\{-\left(\left(\mathrm{a}_{4} \lambda_{321}+\left(1-\mathrm{a}_{4}\right) \lambda_{320}\right)+\left(\mathrm{a}_{8} \mu_{321}+\left(1-\mathrm{a}_{8}\right) \mu_{320}\right)+\left(\mathrm{a}_{9} \delta_{321}+\left(1-\mathrm{a}_{9}\right) \delta_{320}\right)\right)\right. \\
& \left.+\frac{\left[\left(\mathrm{a}_{8} \mu_{321}+\left(1-\mathrm{a}_{8}\right) \mu_{320}\right)+\left(\mathrm{a}_{9} \delta_{321}+\left(1-\mathrm{a}_{9}\right) \delta_{320}\right)\right]}{\mathrm{e}^{\mathrm{v}}}+\left(\mathrm{a}_{4} \lambda_{321}+\left(1-\mathrm{a}_{4}\right) \lambda_{320}\right) \mathrm{e}^{\mathrm{v}}\right\} \mathrm{k}(\mathrm{u}, \mathrm{v} ; \mathrm{t})
\end{aligned}
$$

Comparing the coefficient of the power of u's and v's in the above equations, we get the following

$$
\begin{aligned}
\frac{\partial}{\partial \mathrm{t}} \mathrm{m}_{1,0}(\mathrm{t})= & \left(\lambda_{1}^{*}-\lambda_{2}^{*}-\mu_{1}^{*}\right) \mathrm{m}_{1,0}(\mathrm{t}) \\
\frac{\partial}{\partial \mathrm{t}} \mathrm{m}_{0,1}(\mathrm{t})= & \lambda_{2}^{*} \mathrm{~m}_{1,0}(\mathrm{t})+\left(\lambda_{3}^{*}-\mu_{2}^{*}-\mu_{3}^{*}\right) \mathrm{m}_{0,1}(\mathrm{t}) \\
\frac{\partial}{\partial \mathrm{t}} \mathrm{m}_{2,0}(\mathrm{t})= & 2\left(\lambda_{1}^{*}-\lambda_{2}^{*}-\mu_{1}^{*}\right) \mathrm{m}_{2,0}(\mathrm{t})+\left(\lambda_{1}^{*}+\lambda_{2}^{*}+\mu_{1}^{*}\right) \mathrm{m}_{1,0}(\mathrm{t}) \\
\frac{\partial}{\partial \mathrm{t}} \mathrm{m}_{0,2}(\mathrm{t})= & \lambda_{2}^{*} \mathrm{~m}_{1,0}(\mathrm{t})+\left(\lambda_{3}^{*}+\mu_{2}^{*}+\mu_{3}^{*}\right) \mathrm{m}_{0,1}(\mathrm{t})+2\left(\lambda_{3}^{*}-\mu_{2}^{*}-\mu_{3}^{*}\right) \mathrm{m}_{0,2}(\mathrm{t}) \\
& +2\left(\lambda_{4}^{*}-\mu_{4}^{*}-\mu_{5}^{*}\right) \mathrm{m}_{0,1}(\mathrm{t})+\lambda_{2}^{*} \mathrm{~m}_{1,1}(\mathrm{t}) \\
\frac{\partial}{\partial \mathrm{t}} \mathrm{m}_{1,1}(\mathrm{t})= & \left(\lambda_{1}^{*}-\lambda_{2}^{*}-\mu_{1}^{*}\right) \mathrm{m}_{1,1}(\mathrm{t})+\lambda_{2}^{*} \mathrm{~m}_{2,0}(\mathrm{t})-\lambda_{2}^{*} \mathrm{~m}_{1,0}(\mathrm{t})+\left(\lambda_{3}^{*}-\mu_{2}^{*}-\mu_{3}^{*}\right) \mathrm{m}_{1,1}(\mathrm{t}) \\
& +\left(\lambda_{4}^{*}-\mu_{4}^{*}-\mu_{5}^{*}\right) \mathrm{m}_{1,0}(\mathrm{t})+\lambda_{2}^{*} \mathrm{~m}_{2,0}(\mathrm{t})
\end{aligned}
$$

Let $m_{i, j}(t)$ denotes the moments of order (i,j) of the normal cells, mutant cells in an organ at time t. Then the characteristics of the model are obtained by solving the above ordinary linear differential equations, which are as follows

Expected number of normal cells in an organ at time ' $t$ '

$\mathrm{m}_{1,0}(\mathrm{t})=\mathrm{N}_{0} \mathrm{e}^{\mathrm{At}}$

Expected number of mutant cells in an organ at time ' $\mathrm{t}$ '

$\mathrm{m}_{0,1}(\mathrm{t})=\frac{\lambda_{2}^{*} \mathrm{~N}_{0} \mathrm{e}^{\mathrm{At}}}{\mathrm{A}-\mathrm{B}}+\left(\mathrm{M}_{0}-\frac{\lambda_{2}^{*} \mathrm{~N}_{0}}{\mathrm{~A}-\mathrm{B}}\right) \mathrm{e}^{\mathrm{Bt}}$

Variance of number of normal cells in the organ at time' $\mathrm{t}$ '

$\mathrm{m}_{2,0}(\mathrm{t})=\frac{\mathrm{DN}_{0} \mathrm{e}^{\mathrm{At}}}{\mathrm{A}}\left(\mathrm{e}^{\mathrm{At}}-1\right)$

Variance of number of mutant cells in the organ at time ' $\mathrm{t}$ '

$$
\begin{aligned}
& \mathrm{m}_{0,2}(\mathrm{t})=\frac{\lambda_{2}^{*} \mathrm{~N}_{0} \mathrm{e}^{\mathrm{At}}}{\mathrm{A}-2 \mathrm{~B}}+(\mathrm{F}+2 \mathrm{E})\left\{\frac{\lambda_{2}^{*} \mathrm{~N}_{0} \mathrm{e}^{\mathrm{At}}}{(\mathrm{A}-2 \mathrm{~B})(\mathrm{A}-\mathrm{B})}-\left(\mathrm{M}_{0}-\frac{\lambda_{2}^{*} \mathrm{~N}_{0}}{\mathrm{~A}-\mathrm{B}}\right) \frac{\mathrm{e}^{\mathrm{Bt}}}{\mathrm{B}}\right\} \\
& +\lambda_{2}^{*}\left\{\begin{array}{l}
\frac{\lambda_{2}^{*} N_{0} D}{A}\left(\frac{e^{2 A t}}{2(A-B)^{2}}+\frac{e^{A t}}{(A-2 B) B}\right)-\frac{\left(E-\lambda_{2}^{*}\right) N_{0} e^{A t}}{(A-2 B) B} \\
-\left(\frac{\lambda_{2}^{*} N_{0} D-(A-B)\left(E-\lambda_{2}^{*}\right) N_{0}}{(A-B) B}\right) \frac{e^{(A+B) t}}{(A-B)}
\end{array}\right\} \\
& -\left\{\begin{array}{l}
\frac{\lambda_{2}^{*} \mathrm{~N}_{0}}{(\mathrm{~A}-2 \mathrm{~B})}+(\mathrm{F}+2 \mathrm{E})\left(\frac{\lambda_{2}^{*} \mathrm{~N}_{0}-(\mathrm{A}-2 \mathrm{~B}) \mathrm{M}_{0}}{2(\mathrm{~A}-2 \mathrm{~B})(\mathrm{A}-\mathrm{B})^{2} \mathrm{~B}}\right) \\
+\lambda_{2}^{* 2} \mathrm{~N}_{0}\left(\frac{2(\mathrm{~A}-\mathrm{B})\left(\lambda_{2}^{*}-\mathrm{E}\right)+\mathrm{D} \lambda_{2}^{*}}{2(\mathrm{~A}-2 \mathrm{~B})(\mathrm{A}-\mathrm{B})^{2}}\right)
\end{array}\right\} \mathrm{e}^{2 \mathrm{Bt}}
\end{aligned}
$$

Covariance of number of normal and mutant cells in an organ at time ' $\mathrm{t}$ ' 
$\mathrm{m}_{1,1}(\mathrm{t})=\left\{\frac{\left(\mathrm{E}-\lambda_{2}^{*}\right) \mathrm{N}_{0} \mathrm{e}^{-\mathrm{Bt}}}{-\mathrm{B}}+\frac{\lambda_{2}^{*} \mathrm{DN}_{0}}{\mathrm{~A}}\left(\frac{\mathrm{e}^{2 \mathrm{At}}}{(\mathrm{A}-\mathrm{B})}+\frac{\mathrm{e}^{\mathrm{At}}}{\mathrm{B}}\right)\right\}+\left\{\frac{\lambda_{2}^{*} \mathrm{DN} \mathrm{N}_{0}-(\mathrm{A}-\mathrm{B})\left(\mathrm{E}-\lambda_{2}^{*}\right) \mathrm{N}_{0}}{(\mathrm{~A}-\mathrm{B}) \mathrm{B}}\right\} \mathrm{e}^{(\mathrm{A}+\mathrm{B}) \mathrm{t}}$

Where, $\mathrm{N}_{0} \& \mathrm{M}_{0}$ - Initial number of normal and mutant cells in an organ
$\mathrm{A}=\lambda_{1}^{*}-\lambda_{2}^{*}-\mu_{1}^{*}$
$\mathrm{B}=\lambda_{3}^{*}-\mu_{2}^{*}-\mu_{3}^{*}$
$\mathrm{D}=\lambda_{1}^{*}+\lambda_{2}^{*}+\mu_{1}^{*}$
$\mathrm{E}=\lambda_{4}^{*}-\mu_{4}^{*}-\mu_{5}^{*}$
$\mathrm{F}=\lambda_{3}^{*}+\mu_{2}^{*}+\mu_{3}^{*}$
$\lambda_{1}^{*}=\mathrm{a}_{1} \lambda_{111}+\left(1-\mathrm{a}_{1}\right) \lambda_{110}$
$\lambda_{2}^{*}=a_{2} \delta_{111}+\left(1-a_{2}\right) \delta_{110}$
$\lambda_{3}^{*}=a_{3} \lambda_{211}+\left(1-a_{3}\right) \lambda_{210}$
$\lambda_{4}^{*}=\mathrm{a}_{4} \lambda_{321}+\left(1-\mathrm{a}_{4}\right) \lambda_{320}$
$\mu_{1}^{*}=a_{5} \mu_{111}+\left(1-a_{5}\right) \mu_{110}$
$\mu_{2}^{*}=a_{6} \mu_{211}+\left(1-a_{6}\right) \mu_{210}$
$\mu_{3}^{*}=a_{7} \delta_{211}+\left(1-a_{7}\right) \delta_{210}$
$\mu_{4}^{*}=a_{8} \mu_{321}+\left(1-a_{8}\right) \mu_{320}$
$\mu_{5}^{*}=a_{9} \delta_{321}+\left(1-a_{9}\right) \delta_{320}$

\section{Numerical Illustration}

The computed values of the characteristics of the model $\mathrm{m}_{1,0}(\mathrm{t}), \mathrm{m}_{0,1}(\mathrm{t}), \mathrm{m}_{2,0}(\mathrm{t}), \mathrm{m}_{0,2}(\mathrm{t})$ and $\mathrm{m}_{1,1}(\mathrm{t})$ mentioned above from equation (3.9) to (3.13) for the parameters are presented in the tables for changing values of $\lambda_{111}, \lambda_{110}, \delta_{111}, \delta_{110}, \lambda_{211}, \lambda_{210}, \lambda_{321}, \lambda_{320}, \mu_{111}, \mu_{110}, \mu_{211}, \mu_{210}, \delta_{211}, \delta_{210}, \mu_{321}, \mu_{320}, \delta_{321}, \delta_{320}$ and $t$ in the appendix-I. The linear function is defined to connection the kinetics of cells in the tumor under presence and vacation period of drug therapy.

\section{Findings}

The findings were made by changing one decision parameter while fixing other parameters are constant.

- $\mathrm{m}_{10,}, \mathrm{~m}_{01}, \mathrm{~m}_{20}, \mathrm{~m}_{02}$ and $\mathrm{m}_{11}$ are the increasing function of initial size normal cells $\mathrm{N}_{0}$.

- $\mathrm{m}_{10}, \mathrm{~m}_{20}, \mathrm{~m}_{11}$ are invariant and $\mathrm{m}_{01}, \mathrm{~m}_{02}$ are increasing function of initial number of mutant cells $\mathrm{M}_{0}$.

- $\mathrm{m}_{10}, \mathrm{~m}_{01}, \mathrm{~m}_{20}, \mathrm{~m}_{02}$ and $\mathrm{m}_{11}$ are the increasing function arrival of normal cells $\lambda_{111}$ and $\lambda_{110}$.

- $\mathrm{m}_{10}, \mathrm{~m}_{20}, \mathrm{~m}_{02}$ and $\mathrm{m}_{11}$ are decreasing and $\mathrm{m}_{01}$ is an increasing function of transformation rate of normal cells to mutant cells $\delta_{111}$ and $\delta_{110}$.

- $\mathrm{m}_{10,} \mathrm{~m}_{20}$ are invariant and, $\mathrm{m}_{01}, \mathrm{~m}_{02}$ and $\mathrm{m}_{11}$ are the increasing function arrival of mutant cells $\lambda_{211}$ and $\lambda_{210}$.

- $\mathrm{m}_{10}, \mathrm{~m}_{01}, \mathrm{~m}_{20}$ are invariant and, $\mathrm{m}_{02}$ and $\mathrm{m}_{11}$ are the increasing function growth rate of mutant cells in secondary tumor $\lambda_{321}$ and $\lambda_{320}$.

- $\mathrm{m}_{10,}, \mathrm{~m}_{01}, \mathrm{~m}_{20}, \mathrm{~m}_{02}$ and $\mathrm{m}_{11}$ are decreasing function of death rate of normal cells $\mu_{111}$ and $\mu_{110}$.

- $\mathrm{m}_{10}, \mathrm{~m}_{20}$ are invariant and $\mathrm{m}_{01}, \mathrm{~m}_{02}$ and $\mathrm{m}_{11}$ are decreasing function death rate of mutant cells $\mu_{211}$ and $\mu_{210}$.

- $\mathrm{m}_{10}, \mathrm{~m}_{01}, \mathrm{~m}_{20}$ are invariant and, $\mathrm{m}_{02}$ and $\mathrm{m}_{11}$ are the decreasing function migration rate of mutant cells to in secondary tumor $\delta_{211}$ and $\delta_{210}$.

- $\mathrm{m}_{10}, \mathrm{~m}_{01}, \mathrm{~m}_{20}$ are invariant and, $\mathrm{m}_{02}$ and $\mathrm{m}_{11}$ are the decreasing function death rate of mutant cells in secondary tumor $\mu_{321}$ and $\mu_{320}$.

- $\mathrm{m}_{10}, \mathrm{~m}_{01}, \mathrm{~m}_{20}$ are invariant and, $\mathrm{m}_{02}$ and $\mathrm{m}_{11}$ are the decreasing function migration rate of mutant cells in secondary tumor $\mu_{321}$ and $\mu_{320}$.

- $\mathrm{m}_{10}, \mathrm{~m}_{01}, \mathrm{~m}_{20}, \mathrm{~m}_{02}$ and $\mathrm{m}_{11}$ are the increasing function of time $\mathrm{t}$.

The above findings are describing dynamics of the measures derived from the developed stochastic model and $a_{k}$ is assumed as the partial presence of drug.

\section{Acknowledgements:}

The authors are thankful to acknowledge the funding agency to extract this study as the first author is the principal investigator of a major research project work entitled "Studies on stochastic models for cancer growth and its application to optimal drug administration with chemotherapy" sponsored by the Scientific and Engineering Research Board (SERB), Department of Science \& Technology (DST), Govt. of India.

\section{References}

[1] Iwata K, Kawasaki K, Shigesasa N (2000) A dynamical model for the growth and size distribution of multiple metastasis tumors. Jounal. Theor. Biol., 203: 177-186

[2] Pinho S T R, Freedman H I, Nani F (2002) A Chemotherapy model for the treatment of cancer with metastasis. Mathematical and Computer Modelling, 36: 773-803 
Stochastic Modelling of Tumor Growth within Organ during Chemotherapy Using Bivariate Birth,

[3] Srinivasa Rao K, Tirupathi Rao P (2004) A Stochastic Model for Cancer Cell Growth under Chemotherapy. Assam Statistical Review. 18(1): 81-101

[4] Srinivasa Rao K, Tirupathi Rao P (2004) Stochastic Model for Mutant Cell Growth with Inactivation of Allele Genes. Journal of Statistical Theory and Applications. 3(1): 75-85

[5] Tirupathi Rao P, Madhavi K, Masthan Babu S K (2011) Bivariate Stochastic Modeling for Mutant Cell Growth under Chemotherapy. International Journal of Mathematics and applications. 4(1): 1-12

[6] Tirupathi Rao P, Naveen Kumar B N, Reddy P R S (2013) Three Stage Stochastic Modelling for Cancer cell growth under chemotherapy. International Journal of Advanced Computer and Mathematical Sciences. 4(2): 168-180

[7] Tirupathi Rao P, Srinivasa Rao K (2004) Stochastic Model for Cancer Cell Growth with Spontaneous Mutation and Proliferation. International Journal of Management and Systems. 20(1): 85-93

Appendix-1: Table for all statistical measures with varying values of one parameter when other parameters are fixed

\begin{tabular}{|c|c|c|c|c|c|c|c|c|c|c|c|c|c|c|c|c|c|c|c|c|c|c|c|c|}
\hline$x_{0}$ & Mto & 2 & 3 & 3 & 3 & $20=1$ & 30 & 30 & $3 \mathrm{me}$ & $\mathrm{lm}$ & $\mathrm{Ba}$ & Bum & $\Delta \mathrm{man}$ & 3... & b. & $\mathrm{Bm}$ & $\mathrm{hm}$ & 3 & 3 & $m_{4}$ & $m_{4}$ & $m_{14}$ & $m_{i 4}$ & $\mathrm{~m}$ \\
\hline 3000 & 50 & & & 00 & -1 & & & 0.1 & 0.2 & 01 & \begin{tabular}{|l|}
05 \\
\end{tabular} & & \begin{tabular}{|l|l} 
\\
\end{tabular} & 0.01 & 02 & 0.1 & \begin{tabular}{|l|}
0.00 \\
\end{tabular} & 0001 & 0.01 & $\cos .919$ & 650 & 105 & 11110 & $\cos 2$ \\
\hline$\infty$ & 50 & & & 001 & 01 & & 4 & 01 & \begin{tabular}{|l|l}
03 \\
\end{tabular} & 0.1 & \begin{tabular}{|l|}
05 \\
\end{tabular} & 1 & \begin{tabular}{|l|}
05 \\
\end{tabular} & \begin{tabular}{|l|}
0.01 \\
\end{tabular} & \begin{tabular}{|l|l}
03 \\
\end{tabular} & 01 & \begin{tabular}{|l|}
000 \\
\end{tabular} & 0.001 & 001 & $m, 0 n$ & 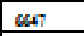 & \begin{tabular}{|l|l}
1979 \\
\end{tabular} & He:0 & $a x+4$ \\
\hline$s 00$ & 50 & & & 001 & 01 & & 4 & 01 & \begin{tabular}{|l|l} 
\\
\end{tabular} & 0.1 & \begin{tabular}{|l|} 
\\
\end{tabular} & 1 & \begin{tabular}{|l|}
05 \\
\end{tabular} & \begin{tabular}{|l|}
001 \\
\end{tabular} & \begin{tabular}{|l|}
03 \\
\end{tabular} & 01 & \begin{tabular}{|l|}
00 \\
\end{tabular} & 0001 & 001 & 1008 & $\cos 5$ & \begin{tabular}{|l|l|}
2654 \\
\end{tabular} & $117 \times 0$ & 50.053 \\
\hline 000 & 0 & & & 0.01 & 0.1 & & 4 & 0.1 & 02 & 01 & \begin{tabular}{|l|}
05 \\
\end{tabular} & 1 & \begin{tabular}{|l|l}
$0 s$ \\
\end{tabular} & \begin{tabular}{|l|l} 
\\
\end{tabular} & 0 & 0.1 & \begin{tabular}{|l|}
000 \\
\end{tabular} & 0.001 & 0 & 1206 & $\pi s$ & 3000 & 12000 & $20 \times 0,6$ \\
\hline 7000 & 50 & 2 & & 001 & 01 & & 8 & 01 & \begin{tabular}{|l|}
03 \\
\end{tabular} & 01 & 05 & 1 & \begin{tabular}{|l|}
05 \\
\end{tabular} & \begin{tabular}{|l|l|} 
\\
\end{tabular} & \begin{tabular}{|l|}
03 \\
\end{tabular} & 01 & \begin{tabular}{|l|}
000 \\
\end{tabular} & 0001 & 001 & 1007 & $m$ & 304 & 100 & 1116 \\
\hline 200 & 290 & 2 & & 000 & 01 & & 4 & 0.1 & \begin{tabular}{|l|l} 
\\
\end{tabular} & 01 & \begin{tabular}{|l|}
05 \\
\end{tabular} & 1 & \begin{tabular}{|l|}
05 \\
\end{tabular} & \begin{tabular}{|l|}
0.01 \\
\end{tabular} & 0 & \begin{tabular}{|l|} 
\\
\end{tabular} & \begin{tabular}{|l|}
000 \\
\end{tabular} & 0.001 & 001 & -01.965 & 767 & 200.60 & $110=0$ & $\mathrm{~m} 2 \mathrm{~m}$ \\
\hline 200 & 600 & & & \begin{tabular}{|l|l|}
000 \\
\end{tabular} & 01 & & 4 & 01 & \begin{tabular}{|l|l}
03 \\
\end{tabular} & 0.1 & \begin{tabular}{|l|l}
04 \\
\end{tabular} & 1 & \begin{tabular}{|l|l}
05 \\
\end{tabular} & \begin{tabular}{|l|l}
001 \\
\end{tabular} & 0 & 01 & \begin{tabular}{|l|l|}
000 \\
\end{tabular} & 0.001 & 00 & -01.964 & 786 & 200.60 & 1000 & $\mathrm{mim}$ \\
\hline 20 & 60 & 3 & & 008 & 0.1 & & 4 & 01 & \begin{tabular}{|l|}
03 \\
\end{tabular} & 0.1 & \begin{tabular}{|l|}
05 \\
\end{tabular} & 1 & \begin{tabular}{|l|}
05 \\
\end{tabular} & \begin{tabular}{|l|}
0.01 \\
\end{tabular} & 0 & 01 & \begin{tabular}{|l|}
000 \\
\end{tabular} & 0,007 & 001 & 01.96 & $\operatorname{sen}$ & 900.68 & 1000 & $\mathrm{~mm}$ \\
\hline 200 & 7000 & 2 & & 001 & 01 & & 4 & 0.1 & \begin{tabular}{|l|l} 
\\
\end{tabular} & 0.1 & \begin{tabular}{|l|}
05 \\
\end{tabular} & 1 & \begin{tabular}{|l|}
05 \\
\end{tabular} & \begin{tabular}{|l|}
0.01 \\
\end{tabular} & \begin{tabular}{|l|}
03 \\
\end{tabular} & \begin{tabular}{|l|}
01 \\
\end{tabular} & \begin{tabular}{|l|}
000 \\
\end{tabular} & 0.001 & \begin{tabular}{|l|l|}
0 \\
\end{tabular} & -01.964 & 933 & 920.68 & 1070 & mam \\
\hline 200 & 790 & & & 001 & 01 & & 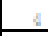 & 01 & \begin{tabular}{|l|l}
03 \\
\end{tabular} & 01 & \begin{tabular}{|l|l}
04 \\
\end{tabular} & 1 & \begin{tabular}{|l|l}
$0 s$ \\
\end{tabular} & \begin{tabular}{|l|l} 
\\
\end{tabular} & \begin{tabular}{|l|l}
03 \\
\end{tabular} & 01 & \begin{tabular}{|l|}
000 \\
\end{tabular} & 0,001 & \begin{tabular}{|l|}
001 \\
\end{tabular} & -01.96 & $\mathrm{mmo}$ & 900.60 & 10000 & $\mathrm{man}$ \\
\hline 200 & 500 & 21 & & 001 & 01 & & - & 0.1 & \begin{tabular}{|l|}
03 \\
\end{tabular} & 01 & \begin{tabular}{|l|}
05 \\
\end{tabular} & 1 & \begin{tabular}{|l|}
05 \\
\end{tabular} & \begin{tabular}{|l|}
001 \\
\end{tabular} & \begin{tabular}{|l|}
03 \\
\end{tabular} & 0.1 & \begin{tabular}{|l|}
000 \\
\end{tabular} & 0,001 & \begin{tabular}{|l|l|}
001 \\
\end{tabular} & 40.066 & 6582 & \begin{tabular}{|l|l|}
1008 \\
\end{tabular} & 1120 & ienou \\
\hline 200 & 50 & 2.2 & & 001 & 01 & & 4 & 01 & 03 & 0. & 0.5 & 1 & \begin{tabular}{|l|}
05 \\
\end{tabular} & \begin{tabular}{|l|l|} 
\\
\end{tabular} & 00 & \begin{tabular}{|l|l} 
\\
\end{tabular} & \begin{tabular}{|l|l|} 
\\
\end{tabular} & 0.001 & \begin{tabular}{|l|l} 
\\
\end{tabular} & $418: 6$ & 652 & \begin{tabular}{|l|l|} 
\\
\end{tabular} & $111 \times$ & in.s. \\
\hline 20 & \begin{tabular}{|l|l}
50 \\
\end{tabular} & 2. & & 001 & 01 & & - & 01 & \begin{tabular}{|l|l}
03 \\
\end{tabular} & 01 & \begin{tabular}{|l|}
05 \\
\end{tabular} & 1 & \begin{tabular}{|l|l}
$0 s$ \\
\end{tabular} & \begin{tabular}{|l|}
001 \\
\end{tabular} & \begin{tabular}{|l|}
03 \\
\end{tabular} & 01 & \begin{tabular}{|l|}
000 \\
\end{tabular} & 0,001 & \begin{tabular}{|l|l}
001 \\
\end{tabular} & $\Leftrightarrow a .001$ & $6 s 5$ & 1118 & 11000 & 促: 81 \\
\hline 200 & 800 & 2.4 & & 008 & 01 & & 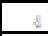 & 01 & \begin{tabular}{|l|}
03 \\
\end{tabular} & 0.1 & 05 & 1 & \begin{tabular}{|l|}
05 \\
\end{tabular} & \begin{tabular}{|l|l|}
0.01 \\
\end{tabular} & 08 & 0.1 & \begin{tabular}{|l|}
000 \\
\end{tabular} & 0.001 & 001 & 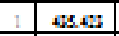 & $\cos 4$ & 1174 & 1280 & क67.28 \\
\hline 200 & 5 & 2.5 & & 001 & 01 & & - & 01 & \begin{tabular}{|l|l}
03 \\
\end{tabular} & 01 & \begin{tabular}{|l|}
05 \\
\end{tabular} & 1 & \begin{tabular}{|l|}
05 \\
\end{tabular} & \begin{tabular}{|l|} 
\\
\end{tabular} & \begin{tabular}{|l|}
03 \\
\end{tabular} & \begin{tabular}{|l|l} 
\\
\end{tabular} & \begin{tabular}{|l|l|}
000 \\
\end{tabular} & 0.001 & 001 & 世2:19 & $\operatorname{coss}$ & $1 \mathrm{~s}$ & $1 w 0$ & \begin{tabular}{|l|l|}
$n \in R e$ \\
\end{tabular} \\
\hline 200 & 50 & 2 & 1.2 & 001 & 01 & - & 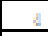 & 01 & \begin{tabular}{|l|l}
03 \\
\end{tabular} & 01 & \begin{tabular}{|l|}
05 \\
\end{tabular} & 1 & \begin{tabular}{|l|l}
$0 s$ \\
\end{tabular} & \begin{tabular}{|l|}
001 \\
\end{tabular} & \begin{tabular}{|l|l}
03 \\
\end{tabular} & 01 & \begin{tabular}{|l|}
000 \\
\end{tabular} & 0,001 & 001 & fl. 1.08 & 6057 & 1991 & 15170 & exess \\
\hline 200 & 800 & & Lis & 004 & 01 & & - & 0.1 & \begin{tabular}{|l|}
03 \\
\end{tabular} & 0.1 & 05 & 1 & \begin{tabular}{|l|}
05 \\
\end{tabular} & 0.01 & \begin{tabular}{|l|}
03 \\
\end{tabular} & 0.1 & \begin{tabular}{|l|}
000 \\
\end{tabular} & 0001 & 0.01 & s10.97 & 6000 & 1685 & 17000 & $40.5 \mathrm{sm}$ \\
\hline 20 & 50 & & $L .4$ & 001 & 01 & & -4 & 01 & \begin{tabular}{|l|l} 
\\
\end{tabular} & 01 & \begin{tabular}{|l|}
05 \\
\end{tabular} & 1 & \begin{tabular}{|l|}
05 \\
\end{tabular} & \begin{tabular}{|l|l|} 
\\
\end{tabular} & \begin{tabular}{|l|}
03 \\
\end{tabular} & \begin{tabular}{|l|} 
\\
\end{tabular} & \begin{tabular}{|c|}
00 \\
\end{tabular} & 0.001 & \begin{tabular}{|l|l}
001 \\
\end{tabular} & sans & $\omega i$ & \begin{tabular}{|l|l|}
196 \\
\end{tabular} & 50000 & es:os: \\
\hline 200 & 500 & 2 & \begin{tabular}{|l|}
1.5 \\
\end{tabular} & 008 & 01 & 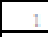 & - & 01 & \begin{tabular}{|l|l}
03 \\
\end{tabular} & 01 & \begin{tabular}{|l|}
05 \\
\end{tabular} & 1 & \begin{tabular}{|l|}
05 \\
\end{tabular} & \begin{tabular}{|l|}
001 \\
\end{tabular} & \begin{tabular}{|l|}
03 \\
\end{tabular} & \begin{tabular}{|l|}
01 \\
\end{tabular} & \begin{tabular}{|l|}
000 \\
\end{tabular} & 0007 & 001 & 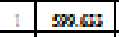 & 6006 & 204 & 20620 & ss.m: \\
\hline 700 & 50 & 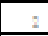 & 1.6 & 001 & 0.1 & & & 01 & \begin{tabular}{|l|}
03 \\
\end{tabular} & 01 & \begin{tabular}{|l|}
05 \\
\end{tabular} & -1 & \begin{tabular}{|l|}
04 \\
\end{tabular} & 0.01 & \begin{tabular}{|l|}
03 \\
\end{tabular} & 0.1 & \begin{tabular}{|l|}
000 \\
\end{tabular} & 0001 & \begin{tabular}{|l|l|}
0.1 \\
\end{tabular} & 60.54 & 6570 & 2705 & $=0000$ & 611.857 \\
\hline 200 & 50 & & & 0.02 & 01 & & 4 & 0.1 & \begin{tabular}{|l|l}
03 \\
\end{tabular} & 0.1 & \begin{tabular}{|l|}
05 \\
\end{tabular} & 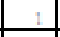 & \begin{tabular}{|l|l}
05 \\
\end{tabular} & \begin{tabular}{|l|l}
00 \\
\end{tabular} & \begin{tabular}{|l|}
03 \\
\end{tabular} & 0.1 & \begin{tabular}{|l|l|}
0.03 \\
\end{tabular} & 0.001 & \begin{tabular}{|l|l|} 
\\
\end{tabular} & $\cos m$ & का & 2000 & $20 x=0$ & $610 \mathrm{~ms}$ \\
\hline 200 & 500 & 3 & & 0.04 & 01 & 1 & 4 & 01 & \begin{tabular}{|l|l}
03 \\
\end{tabular} & 01 & \begin{tabular}{|l|}
05 \\
\end{tabular} & 1 & \begin{tabular}{|l|}
05 \\
\end{tabular} & \begin{tabular}{|l|l|}
0.01 \\
\end{tabular} & \begin{tabular}{|l|l}
03 \\
\end{tabular} & 0.1 & \begin{tabular}{|l|}
000 \\
\end{tabular} & 0,001 & 001 & $\cos c 0$ & 6570 & \begin{tabular}{|l|l|}
2006 \\
\end{tabular} & $\operatorname{soc} 0$ & con:sos \\
\hline 200 & 800 & 3 & & 0.06 & 01 & & - & 0.1 & \begin{tabular}{|l|}
03 \\
\end{tabular} & 01 & \begin{tabular}{|l|}
05 \\
\end{tabular} & 1 & \begin{tabular}{|l|}
05 \\
\end{tabular} & 0.01 & \begin{tabular}{|l|}
02 \\
\end{tabular} & 01 & \begin{tabular}{|l|}
0.00 \\
\end{tabular} & 0001 & \begin{tabular}{|l|l|}
001 \\
\end{tabular} & Ge.1.1 & 60 & 2074 & 2340 & $\cos 70$ \\
\hline 200 & 500 & & & 0.00 & 01 & & 4 & 01 & 02 & 0.1 & \begin{tabular}{|l|}
04 \\
\end{tabular} & 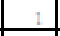 & \begin{tabular}{|l|l}
$0 s$ \\
\end{tabular} & \begin{tabular}{|l|l} 
\\
\end{tabular} & \begin{tabular}{|l|l|} 
\\
\end{tabular} & 0.1 & \begin{tabular}{|l|}
000 \\
\end{tabular} & 0.001 & \begin{tabular}{|l|l|} 
\\
\end{tabular} & $\cos 24$ & 600 & \begin{tabular}{|l|l|}
2622 \\
\end{tabular} & 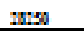 & (61.017 \\
\hline 700 & 50 & 3 & & 0.1 & 01 & 1 & 4 & 01 & \begin{tabular}{|l|l|}
03 \\
\end{tabular} & 0.1 & \begin{tabular}{|l|}
05 \\
\end{tabular} & 1 & \begin{tabular}{|l|}
05 \\
\end{tabular} & \begin{tabular}{|l|}
001 \\
\end{tabular} & \begin{tabular}{|l|}
03 \\
\end{tabular} & \begin{tabular}{|l|}
01 \\
\end{tabular} & \begin{tabular}{|l|}
000 \\
\end{tabular} & 0.001 & 001 & an.287 & 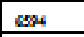 & \begin{tabular}{|l|l}
2650 \\
\end{tabular} & 20000 & $\operatorname{sen} 117$ \\
\hline 200 & 500 & 2 & & 000 & 0.2 & & 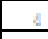 & 01 & \begin{tabular}{|l|l}
03 \\
\end{tabular} & 01 & \begin{tabular}{|l|}
04 \\
\end{tabular} & 1 & \begin{tabular}{|l|l}
$0 s$ \\
\end{tabular} & \begin{tabular}{|l|}
0.01 \\
\end{tabular} & \begin{tabular}{|l|l}
03 \\
\end{tabular} & \begin{tabular}{|l|}
0.1 \\
\end{tabular} & \begin{tabular}{|l|}
000 \\
\end{tabular} & 0,001 & \begin{tabular}{|l|l}
001 \\
\end{tabular} & 50.00 & 6cts -25 & $2,672-15$ & $25 \pi-4$ & sest2 \\
\hline 200 & \begin{tabular}{|l|l}
50 \\
\end{tabular} & & & \begin{tabular}{|l|l|}
000 \\
\end{tabular} & 0.5 & & 4 & 04 & \begin{tabular}{|l|l}
03 \\
\end{tabular} & 0.1 & \begin{tabular}{|l|l}
04 \\
\end{tabular} & 1 & \begin{tabular}{|l|l}
05 \\
\end{tabular} & \begin{tabular}{|l|}
0.01 \\
\end{tabular} & \begin{tabular}{|l|l}
03 \\
\end{tabular} & 0.1 & \begin{tabular}{|l|}
000 \\
\end{tabular} & 0.001 & 001 & s.2.5n & $6 \pi \pi-15$ & $2: 5 \pi-45$ & $22 \pi 4-4$ & $306 c_{2}$ \\
\hline 200 & 50 & 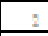 & & 008 & 0.4 & 1 & 4 & 0.1 & \begin{tabular}{|l|l|}
02 \\
\end{tabular} & 0.1 & \begin{tabular}{|l|}
05 \\
\end{tabular} & 1 & \begin{tabular}{|l|}
05 \\
\end{tabular} & \begin{tabular}{|l|}
0.01 \\
\end{tabular} & 08 & \begin{tabular}{|l|}
01 \\
\end{tabular} & \begin{tabular}{|l|}
000 \\
\end{tabular} & 0.001 & 001 & $510 \mathrm{~m}$ & CGTTEN & $20.05 \pi-45$ & $1.90 \pi-404$ & $455 \mathrm{sc1}$ \\
\hline 200 & 500 & 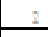 & & 001 & 0.5 & E & 4 & 0.1 & \begin{tabular}{|l|l}
01 \\
\end{tabular} & 01 & \begin{tabular}{|l|}
05 \\
\end{tabular} & 1 & \begin{tabular}{|l|l}
05 \\
\end{tabular} & \begin{tabular}{|l|l|}
0.01 \\
\end{tabular} & \begin{tabular}{|l|}
03 \\
\end{tabular} & \begin{tabular}{|l|}
01 \\
\end{tabular} & \begin{tabular}{|l|}
000 \\
\end{tabular} & 0.001 & \begin{tabular}{|l|l|}
001 \\
\end{tabular} & Af.cos & $698 \pi-08$ & $1.87 \pi-25$ & L.רet-04 & 61.017 \\
\hline 200 & 50 & 2 & & 001 & 0.6 & & 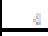 & 0.1 & \begin{tabular}{|l|l}
03 \\
\end{tabular} & 01 & \begin{tabular}{|l|}
04 \\
\end{tabular} & 1 & \begin{tabular}{|l|l}
04 \\
\end{tabular} & \begin{tabular}{|l|l} 
\\
\end{tabular} & \begin{tabular}{|l|l}
03 \\
\end{tabular} & 0.1 & \begin{tabular}{|l|}
000 \\
\end{tabular} & 0.001 & \begin{tabular}{|l|l}
001 \\
\end{tabular} & seses & $7.006-15$ & 1.705-25 & 1.strat & 50.94 \\
\hline 200 & 800 & 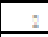 & & 008 & 01 & 1.2 & 4 & 01 & \begin{tabular}{|l|}
03 \\
\end{tabular} & 01 & \begin{tabular}{|l|}
05 \\
\end{tabular} & 1 & \begin{tabular}{|l|}
05 \\
\end{tabular} & \begin{tabular}{|l|l|} 
\\
\end{tabular} & \begin{tabular}{|l|}
03 \\
\end{tabular} & \begin{tabular}{|l|}
01 \\
\end{tabular} & \begin{tabular}{|l|}
000 \\
\end{tabular} & 0001 & \begin{tabular}{|l|l}
001 \\
\end{tabular} & 01.96 & \begin{tabular}{|c|c|}
617 \\
\end{tabular} & 900.68 & 111200 & 20.005 \\
\hline 200 & 50 & 3 & & 001 & 0.1 & 1.5 & 4 & 01 & \begin{tabular}{|l|l}
03 \\
\end{tabular} & 01 & \begin{tabular}{|l|}
05 \\
\end{tabular} & 1 & \begin{tabular}{|l|l}
$0 s$ \\
\end{tabular} & \begin{tabular}{|l|}
0.01 \\
\end{tabular} & 0 & \begin{tabular}{|l|}
01 \\
\end{tabular} & \begin{tabular}{|l|}
000 \\
\end{tabular} & 0,001 & 001 & 50.206 & $\cos _{4}$ & 90.685 & 111200 & iesoln \\
\hline 700 & \begin{tabular}{|l|l}
50 \\
\end{tabular} & 3 & & 001 & 01 & 1.4 & - & 01 & \begin{tabular}{|l|l}
03 \\
\end{tabular} & 01 & \begin{tabular}{|l|}
05 \\
\end{tabular} & 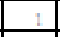 & \begin{tabular}{|l|l}
04 \\
\end{tabular} & \begin{tabular}{|l|}
0.01 \\
\end{tabular} & \begin{tabular}{|l|l}
03 \\
\end{tabular} & \begin{tabular}{|l|}
0.1 \\
\end{tabular} & \begin{tabular}{|l|}
000 \\
\end{tabular} & 0.001 & \begin{tabular}{|l|l}
001 \\
\end{tabular} & -01.968 & $\pi 004$ & 200.60 & 11000 & 30.04 \\
\hline 200 & 50 & 2 & & 001 & 01 & 1.5 & 4 & 01 & \begin{tabular}{|l|}
03 \\
\end{tabular} & 01 & \begin{tabular}{|l|}
05 \\
\end{tabular} & 1 & \begin{tabular}{|l|}
05 \\
\end{tabular} & \begin{tabular}{|l|}
0.01 \\
\end{tabular} & 0 & \begin{tabular}{|l|}
01 \\
\end{tabular} & \begin{tabular}{|l|}
000 \\
\end{tabular} & 0.001 & 001 & 01.96 & 776 & 90.685 & 11670 & wat $\mathrm{DLC}$ \\
\hline 200 & 50 & 3 & & 0.1 & 01 & 1.6 & 4 & 0.1 & \begin{tabular}{|l|}
03 \\
\end{tabular} & 01 & \begin{tabular}{|l|}
05 \\
\end{tabular} & 1 & \begin{tabular}{|l|l}
05 \\
\end{tabular} & \begin{tabular}{|l|}
0.01 \\
\end{tabular} & 03 & 0.1 & \begin{tabular}{|l|}
000 \\
\end{tabular} & 0,001 & 001 & 01.96 & 782 & 200.65 & 110000 & ississ \\
\hline 200 & 50 & 2 & & 000 & 01 & 1 & 4 & 0.1 & \begin{tabular}{|l|l}
03 \\
\end{tabular} & 01 & \begin{tabular}{|l|}
05 \\
\end{tabular} & 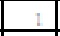 & \begin{tabular}{|l|l}
04 \\
\end{tabular} & \begin{tabular}{|l|}
001 \\
\end{tabular} & \begin{tabular}{|l|}
03 \\
\end{tabular} & \begin{tabular}{|l|}
0.1 \\
\end{tabular} & \begin{tabular}{|l|}
000 \\
\end{tabular} & 0,001 & 001 & -01.96 & 7004 & 200.65 & 11000 & 30.04 \\
\hline 20 & 50 & 2 & & 001 & 01 & & 42 & 01 & \begin{tabular}{|l|}
03 \\
\end{tabular} & 01 & \begin{tabular}{|l|}
05 \\
\end{tabular} & 1 & \begin{tabular}{|l|}
05 \\
\end{tabular} & \begin{tabular}{|l|l|}
001 \\
\end{tabular} & \begin{tabular}{|l|}
09 \\
\end{tabular} & 01 & \begin{tabular}{|l|}
000 \\
\end{tabular} & 0001 & \begin{tabular}{|l|l|}
001 \\
\end{tabular} & 01.26 & 7002 & 280.658 & 1500 & 67.64 \\
\hline 20 & 500 & 2 & & 001 & 01 & & a & 01 & 03 & 0.1 & 05 & 1 & \begin{tabular}{|l|}
05 \\
\end{tabular} & 001 & \begin{tabular}{|l|l} 
\\
\end{tabular} & 0.1 & \begin{tabular}{|c|}
000 \\
\end{tabular} & 0.001 & 0.01 & 01.96 & s.18 & 20.60 & 1300 & 201.014 \\
\hline 700 & 50 & 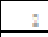 & & 008 & 01 & 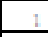 & \begin{tabular}{|l|l}
4 \\
\end{tabular} & 01 & \begin{tabular}{|l|l}
03 \\
\end{tabular} & 01 & \begin{tabular}{|l|}
05 \\
\end{tabular} & - & \begin{tabular}{|l|l}
05 \\
\end{tabular} & \begin{tabular}{|l|}
001 \\
\end{tabular} & \begin{tabular}{|l|}
03 \\
\end{tabular} & \begin{tabular}{|l|}
0.1 \\
\end{tabular} & \begin{tabular}{|l|}
000 \\
\end{tabular} & 0001 & 001 & 01.966 & 2000 & 200.650 & 14000 & - 07.30 \\
\hline 200 & 500 & 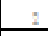 & & \begin{tabular}{|l|l|}
001 \\
\end{tabular} & 0.1 & & \begin{tabular}{|l}
45 \\
\end{tabular} & 01 & \begin{tabular}{|l|l}
03 \\
\end{tabular} & 01 & \begin{tabular}{|l|}
05 \\
\end{tabular} & 1 & \begin{tabular}{|l|}
05 \\
\end{tabular} & 0.01 & 03 & 01 & \begin{tabular}{|l|}
000 \\
\end{tabular} & 0,001 & 001 & 01.94 & r.ss & 200.65 & 15770 & $\leq 0.46$ \\
\hline 200 & 500 & 2 & & \begin{tabular}{|l|l}
001 \\
\end{tabular} & 01 & 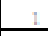 & 4 & $a s$ & \begin{tabular}{|l|l}
03 \\
\end{tabular} & 0.1 & \begin{tabular}{|l|}
05 \\
\end{tabular} & 1 & \begin{tabular}{|l|l}
$0 s$ \\
\end{tabular} & \begin{tabular}{|l|l} 
\\
\end{tabular} & \begin{tabular}{|l|l}
03 \\
\end{tabular} & 0.1 & \begin{tabular}{|l|l|}
003 \\
\end{tabular} & 0,001 & 001 & -01.94 & 6551 & 200.60 & 10000 & -07.00 \\
\hline 200 & 50 & 2 & & 001 & 01 & L & 4 & a.4 & \begin{tabular}{|l|l}
03 \\
\end{tabular} & 01 & \begin{tabular}{|l|}
05 \\
\end{tabular} & - & \begin{tabular}{|l|l}
05 \\
\end{tabular} & 0.01 & \begin{tabular}{|l|}
03 \\
\end{tabular} & \begin{tabular}{|l|}
01 \\
\end{tabular} & \begin{tabular}{|l|}
000 \\
\end{tabular} & 0,001 & 001 & -01.946 & $\omega s 51$ & 200.65 & 10000 & 世4900 \\
\hline 200 & 50 & 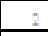 & & \begin{tabular}{|l|l|}
001 \\
\end{tabular} & 01 & & 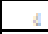 & 0.5 & \begin{tabular}{|l|l}
03 \\
\end{tabular} & 01 & \begin{tabular}{|l|}
05 \\
\end{tabular} & 1 & \begin{tabular}{|l|}
05 \\
\end{tabular} & 0.01 & 0. & 0.1 & \begin{tabular}{|l|}
0.02 \\
\end{tabular} & 0.007 & 0.01 & 01.206 & 6551 & 200.60 & 11170 & 51.98 \\
\hline 200 & 50 & 2 & & \begin{tabular}{|l|l}
001 \\
\end{tabular} & 01 & & - & 0.6 & \begin{tabular}{|l|l} 
\\
\end{tabular} & 01 & 05 & 1 & \begin{tabular}{|l|l}
$0 s$ \\
\end{tabular} & 001 & \begin{tabular}{|l|}
03 \\
\end{tabular} & 0.1 & \begin{tabular}{|l|l|}
000 \\
\end{tabular} & 0.001 & 0.01 & -01.96 & $\omega s 51$ & 250.68 & 11200 & \begin{tabular}{|ll} 
s18:37 \\
\end{tabular} \\
\hline 200 & 50 & 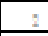 & & 008 & 01 & 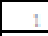 & 4 & 0.7 & \begin{tabular}{|l|l|}
03 \\
\end{tabular} & 01 & \begin{tabular}{|l|}
05 \\
\end{tabular} & - & \begin{tabular}{|l|l}
$0 s$ \\
\end{tabular} & 001 & \begin{tabular}{|l|l}
03 \\
\end{tabular} & \begin{tabular}{|l|}
01 \\
\end{tabular} & \begin{tabular}{|l|}
000 \\
\end{tabular} & 0.001 & 001 & $50.2 \times$ & 6551 & 900.65 & 11250 & $5867 \%$ \\
\hline 20 & 50 & 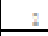 & & 000 & 01 & & 8 & 01 & Q.4 & 0.1 & \begin{tabular}{|l|}
05 \\
\end{tabular} & 1 & \begin{tabular}{|l|}
05 \\
\end{tabular} & \begin{tabular}{|l|l|} 
\\
\end{tabular} & \begin{tabular}{|l|l}
03 \\
\end{tabular} & \begin{tabular}{|l|}
0.1 \\
\end{tabular} & \begin{tabular}{|l|}
000 \\
\end{tabular} & 0,001 & 001 & 01.96 & 6551 & 20.050 & 111170 & 51.99 \\
\hline 200 & 50 & 1 & & \begin{tabular}{|l|l|}
001 \\
\end{tabular} & 01 & 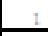 & 8 & 0 & 0.5 & 0.1 & \begin{tabular}{|l|}
05 \\
\end{tabular} & 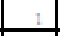 & \begin{tabular}{|l|}
05 \\
\end{tabular} & \begin{tabular}{|l|l} 
\\
\end{tabular} & \begin{tabular}{|l|l}
03 \\
\end{tabular} & 01 & \begin{tabular}{|l|}
000 \\
\end{tabular} & 0.001 & \begin{tabular}{|l|}
001 \\
\end{tabular} & -01.968 & 6551 & 200.65 & $115 \times 0$ & $01.65_{4}$ \\
\hline 200 & 500 & & & 001 & 01 & 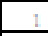 & 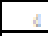 & a & 0.6 & 0 & \begin{tabular}{|l|}
05 \\
\end{tabular} & 1 & \begin{tabular}{|l|l}
05 \\
\end{tabular} & \begin{tabular}{|l|l|} 
\\
\end{tabular} & \begin{tabular}{|l|l}
03 \\
\end{tabular} & \begin{tabular}{|l|}
01 \\
\end{tabular} & \begin{tabular}{|l|}
000 \\
\end{tabular} & 0.001 & 001 & -01.96 & 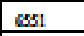 & 200.68 & 11000 & 701.200 \\
\hline 200 & 500 & & & 001 & 0.1 & & 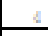 & 0.1 & 0.7 & 0.1 & 05 & 1 & \begin{tabular}{|l|}
05 \\
\end{tabular} & \begin{tabular}{|l|l|} 
\\
\end{tabular} & \begin{tabular}{|l|l} 
\\
\end{tabular} & \begin{tabular}{|l|l} 
\\
\end{tabular} & \begin{tabular}{|l|l|} 
\\
\end{tabular} & 0.001 & \begin{tabular}{|l|l|} 
\\
\end{tabular} & 01.96 & 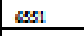 & 250.65 & $1+1+70$ & 21.00s \\
\hline 700 & \begin{tabular}{|l|l}
500 \\
\end{tabular} & - & & \begin{tabular}{|l|l|}
001 \\
\end{tabular} & 01 & - & 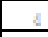 & 01 & 0.8 & 0.1 & \begin{tabular}{|l|}
05 \\
\end{tabular} & 1 & \begin{tabular}{|l|}
05 \\
\end{tabular} & \begin{tabular}{|l|}
0.01 \\
\end{tabular} & \begin{tabular}{|l|l}
07 \\
\end{tabular} & 01 & \begin{tabular}{|l|}
000 \\
\end{tabular} & 0.001 & \begin{tabular}{|l|}
001 \\
\end{tabular} & -01.968 & 6551 & 200.65 & 1060 & 10081 \\
\hline 20 & 50 & & & 0 & 0.1 & 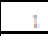 & 4 & 01 & \begin{tabular}{|l|}
03 \\
\end{tabular} & $a:$ : & 05 & 1 & \begin{tabular}{|l|}
04 \\
\end{tabular} & 0 & 0 & \begin{tabular}{|l|}
01 \\
\end{tabular} & \begin{tabular}{|l|}
000 \\
\end{tabular} & 0001 & 001 & 20,185 & 6500 & $981 . \mathrm{mb}$ & 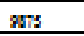 & na:sol \\
\hline 200 & 50 & & & \begin{tabular}{|l|l|}
000 \\
\end{tabular} & 01 & & 4 & 0.1 & \begin{tabular}{|l|l}
03 \\
\end{tabular} & a.4 & 05 & 1 & \begin{tabular}{|l|}
05 \\
\end{tabular} & \begin{tabular}{|l|} 
\\
\end{tabular} & \begin{tabular}{|l|}
03 \\
\end{tabular} & 0.1 & \begin{tabular}{|l|l|}
000 \\
\end{tabular} & 0.001 & \begin{tabular}{|l|l|}
001 \\
\end{tabular} & ness & $\infty 6$ & ma.s. & gest & keso \\
\hline 200 & 50 & - & & 001 & 01 & 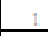 & 4 & 01 & \begin{tabular}{|l|l}
03 \\
\end{tabular} & 0.5 & \begin{tabular}{|l|}
05 \\
\end{tabular} & 1 & \begin{tabular}{|l|}
05 \\
\end{tabular} & \begin{tabular}{|l|l|} 
\\
\end{tabular} & \begin{tabular}{|l|l}
03 \\
\end{tabular} & 01 & \begin{tabular}{|l|}
000 \\
\end{tabular} & 0,001 & \begin{tabular}{|l|}
001 \\
\end{tabular} & moes & 00 & nkm & 2007 & $302 \pi 6$ \\
\hline 200 & \begin{tabular}{|l}
1 \\
\end{tabular} & & & 00 & 01 & & 8 & $a$ & \begin{tabular}{|l|}
03 \\
\end{tabular} & 0.6 & \begin{tabular}{|l|}
05 \\
\end{tabular} & 1 & \begin{tabular}{|l|}
04 \\
\end{tabular} & \begin{tabular}{|l|l}
00 \\
\end{tabular} & 00 & 0 & \begin{tabular}{|l|}
000 \\
\end{tabular} & 000 & 001 & 26.60 & 00 & son.013 & 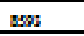 & 20.15 \\
\hline 200 & 50 & & & 00 & $\theta$ & & & 0 & 03 & 0.7 & 05 & & \begin{tabular}{|l|}
05 \\
\end{tabular} & \begin{tabular}{|l|l} 
\\
\end{tabular} & \begin{tabular}{|l|l} 
\\
\end{tabular} & 01 & \begin{tabular}{|c|}
000 \\
\end{tabular} & 0.001 & \begin{tabular}{|l|}
001 \\
\end{tabular} & Whese & $\operatorname{sen}_{0}$ & 572:07 & s19 & 297.65 \\
\hline 700 & \begin{tabular}{|l|l}
500 \\
\end{tabular} & 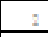 & & 001 & 01 & - & 4 & 01 & \begin{tabular}{|l|l}
03 \\
\end{tabular} & 0 & 0.6 & 1 & \begin{tabular}{|l|}
05 \\
\end{tabular} & \begin{tabular}{|l|l|}
0.01 \\
\end{tabular} & \begin{tabular}{|l|}
03 \\
\end{tabular} & 01 & \begin{tabular}{|l|}
000 \\
\end{tabular} & 0007 & \begin{tabular}{|l|}
001 \\
\end{tabular} & moes & coses & $n<m$ & 2007 & 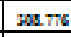 \\
\hline 700 & 30 & & & 0 & 0.1 & 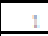 & $g$ & 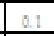 & \begin{tabular}{|l|}
03 \\
\end{tabular} & 0 & 0.7 & 1 & 0.3 & 00 & 08 & 0.1 & \begin{tabular}{|l|}
000 \\
\end{tabular} & 0001 & 00 & Be:516 & 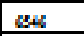 & sents & $7 \mathrm{se}$ & 200600 \\
\hline 200 & $\underline{-2}$ & & & 0 & 0 & & & & $A$ & & \begin{tabular}{|l|} 
\\
\end{tabular} & & 0 & & a & & 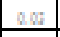 & 0,00 & & $316.18:$ & ses & Treoss & $\omega$ & 36.416 \\
\hline 200 & 50 & 2 & & 001 & 01 & $\overline{-}$ & - & \pm 01 & \begin{tabular}{|l|l}
03 \\
\end{tabular} & 1 & 0.8 & 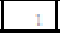 & \begin{tabular}{|l|}
05 \\
\end{tabular} & \begin{tabular}{|l|l|} 
& 0.01 \\
\end{tabular} & \begin{tabular}{|l|l}
03 \\
\end{tabular} & D 01 & \begin{tabular}{|l|l|}
000 \\
\end{tabular} & 0.001 & \begin{tabular}{|l|l|}
0.01 \\
\end{tabular} & 20.07 & $\Delta s t$ & 777.197 & $\pi 00$ & 261.36 \\
\hline
\end{tabular}


Stochastic Modelling of Tumor Growth within Organ during Chemotherapy Using Bivariate Birth,

\begin{tabular}{|c|c|c|c|c|c|c|c|c|c|c|c|c|c|c|c|c|c|c|c|c|c|c|c|c|c|}
\hline No & Mo & $k_{413}$ & $\grave{\lambda}_{310}$ & $\delta_{131}$ & $\delta_{130}$ & $\lambda_{971}$ & $k_{n 38}$ & $\lambda_{n n}$ & $\lambda_{938}$ & $\mu_{3 n}$ & $\mu_{1 x}$ & $\mu_{3 n}$ & $\mu_{2 n}$ & $\delta_{n 31}$ & $\delta_{z a v}$ & $\mu_{2 n}$ & $\mu_{350}$ & $\delta_{3 m}$ & $\delta_{3 z 8}$ & $\mathrm{t}$ & $\mathbf{m}_{10}$ & $\mathbf{m}_{03}$ & $\mathbf{m}_{30}$ & $\mathrm{~m}_{9 z}$ & $\mathbf{m}_{31}$ \\
\hline 300 & 500 & 2 & 1 & 0.01 & 0.1 & 1 & 4 & 0.1 & 0.3 & 0.1 & 0.5 & 1.3 & 0.5 & 0.01 & 0.3 & 0.1 & 0.03 & 0.001 & 0.01 & 1 & 401946 & 6172 & 959.639 & 10530 & 31999 \\
\hline 300 & 300 & 2 & 1 & 0.01 & 0.1 & 1 & 4 & 0.1 & 0.3 & 0.1 & 05 & 1.4 & 05 & 0.01 & 0.3 & 0.1 & 0.03 & 0.001 & 0.01 & 1 & 401945 & 6050 & 959.639 & 10550 & 316.033 \\
\hline 300 & 500 & 2 & 1 & 0.01 & 0.1 & 1 & 4 & 0.1 & 0.3 & 0.1 & 0.5 & 1.5 & 0.5 & 0.01 & 0.3 & 0.1 & 0.03 & 0.001 & 0.01 & 1 & 401945 & 5931 & 959.639 & 10540 & 312.133 \\
\hline 300 & 500 & 2 & 1 & 0.01 & 0.1 & 1 & 4 & 0.1 & 0.3 & 0.1 & 0.5 & 1.6 & 0.5 & 0.01 & 03 & 0.1 & 0.03 & 0.001 & 0.01 & 1 & 401945 & 5814 & 959.639 & 10510 & 306.291 \\
\hline 300 & 500 & 2 & 1 & 0.01 & 0.1 & 1 & 4 & 0.1 & 0.3 & 0.1 & 0.5 & 1 & 0.6 & 0.01 & 03 & 0.1 & 0.03 & 0.001 & 0.01 & 1 & 401946 & 6050 & 959.639 & 10550 & 316.0033 \\
\hline 300 & 500 & 2 & 1 & 0.01 & 0.1 & 1 & 4 & 0.1 & 0.3 & 0.1 & 0.5 & 1 & 0.7 & 0.01 & 0.3 & 0.1 & 0.03 & 0.001 & 0.01 & 1 & 401946 & 5557 & 959.639 & 10470 & 300.39 \\
\hline 300 & 500 & 2 & 1 & 0.01 & 0.1 & 1 & 4 & 0.1 & 0.3 & 0.1 & 0,5 & 1 & 0.8 & 0.01 & 03 & 0.1 & 0.03 & 0.001 & 0.01 & $\begin{array}{ll}3 \\
\end{array}$ & 401946 & 5160 & 959.639 & 10450 & 256.39 \\
\hline 300 & 300 & 2 & 1 & 0.01 & 0.1 & 1 & 4 & 0.1 & 0.3 & 0.1 & 0.5 & 1 & 09 & 0.01 & 03 & 0.1 & 0.03 & 0.001 & 0.01 & 1 & 401946 & 4766 & 959.639 & 10560 & 272.826 \\
\hline 300 & 500 & 2 & 1 & 0.01 & 0.1 & 1 & 4 & 0.1 & 0.3 & 0.1 & 05 & 1 & 1.1 & 0.01 & 03 & 0.1 & 0.03 & 0.001 & 0.01 & 1 & 401946 & 4065 & 959.639 & 11150 & 247958 \\
\hline 300 & 500 & 2 & 1 & 0.01 & 0.1 & 1 & 4 & 0.1 & 0.3 & 0.1 & 0.5 & 1 & 0.5 & 0.04 & 0.3 & 0.1 & 0.03 & 0.001 & 0.01 & 1 & 401946 & 6512 & 959.639 & 10750 & 330.805 \\
\hline 300 & 500 & 2 & 1 & 0.01 & 0.1 & 1 & 4 & 0.1 & 0.3 & 0.1 & 05 & 1 & 05 & 0.06 & 0.3 & 0.1 & 0.03 & 0.001 & 0.01 & 1 & 401946 & 6456 & 959.639 & 10770 & 330.145 \\
\hline 300 & 300 & 2 & 1 & 0.01 & 0.1 & 1 & 4 & 0.1 & 0.3 & 0.1 & 0.5 & 1 & 0.5 & 0.08 & 03 & 0.1 & 0.03 & 0.001 & 0.01 & 1 & 401946 & 6450 & 959.639 & 10750 & 329.319 \\
\hline 300 & 500 & 2 & 1 & 0.01 & 0.1 & 1 & 4 & 0.1 & 0.3 & 0.1 & 0.5 & 1 & 0.5 & 0.1 & 03 & 0.1 & 0.03 & 0.001 & 0.01 & 1 & 401945 & 6435 & 959.639 & 10740 & 328.495 \\
\hline 300 & 300 & 2 & 1 & 0.01 & 0.1 & 1 & 4 & 0.1 & 0.3 & 0.1 & 0.5 & 1 & 0.5 & 0.12 & 03 & 0.1 & 0.03 & 0.001 & 0.01 & 1 & 401945 & 6409 & 959.639 & 10730 & 327.674 \\
\hline 300 & 300 & 2 & 1 & 0.01 & 0.1 & 1 & 4 & 0.1 & 0.3 & 0.1 & 0.5 & 1 & 05 & 0.01 & 0.4 & 0.1 & 0.03 & 0.001 & 0.01 & 1 & 401945 & 6050 & 959.639 & 10550 & 316.033 \\
\hline 300 & 500 & 2 & 1 & 0.01 & 0.1 & 1 & 4 & 0.1 & 0.3 & 0.1 & 0.5 & 1 & 0.5 & 0.01 & 0.5 & 0.1 & 0.03 & 0.001 & 0,01 & 1 & 401945 & 5587 & 959.639 & 10470 & 300.775 \\
\hline 300 & 500 & 2 & 1 & 0.01 & 0.1 & 1 & 4 & 0.1 & 0.3 & 0.1 & 0.5 & 1 & 0.5 & 0.01 & 0.6 & 0.1 & 0.03 & 0.001 & 0.01 & 1 & 401946 & 5160 & 959.639 & 10450 & 286.39 \\
\hline 300 & 500 & 2 & 1 & 0.01 & 0.1 & 1 & 4 & 0.1 & 0.3 & 0.1 & 0.5 & 1 & 0.5 & 0.01 & 0.7 & 0.1 & 0.03 & 0.001 & 0.01 & 1 & 401946 & 4766 & 959.639 & 10560 & 276.826 \\
\hline 300 & 300 & 2 & 1 & 0.01 & 0.1 & 1 & 4 & 0.1 & 0.3 & 0.1 & 0.5 & 1 & 05 & 0.01 & 0.8 & 0.1 & 0.03 & 0.001 & 0.01 & 1 & 401945 & 4402 & 959.639 & 10790 & 260.031 \\
\hline 300 & 500 & 2 & 1 & 0.01 & 0.1 & 1 & 4 & 0.1 & 0.3 & 0.1 & 0.5 & 1 & 0.5 & 0.01 & 03 & 0.3 & 0.03 & 0.001 & 0.01 & 1 & 401946 & 6551 & 959.639 & 10520 & 257.364 \\
\hline 300 & 500 & 2 & 1 & 0.01 & 0.1 & 1 & 4 & 0.1 & 0.3 & 0.1 & 0.5 & 1 & 0.5 & 0.01 & 0.3 & 0.4 & 0.03 & 0.001 & 0.01 & 1 & 401946 & 6551 & 959.639 & 10520 & 219935 \\
\hline 300 & 300 & 2 & 1 & 0.01 & 0.1 & 1 & 4 & 0.1 & 0.3 & 0.1 & 05 & 1 & 05 & 0.01 & 03 & 0.5 & 0.03 & 0.001 & 0.01 & 1 & 401946 & 6551 & 959.639 & 10430 & 152.506 \\
\hline 300 & 500 & 2 & 1 & 0.01 & 0.1 & 1 & 4 & 0.1 & 0.3 & 0.1 & 0.5 & 1 & 0.5 & 0.01 & 0.3 & 0.6 & 0.03 & 0.001 & 0.01 & 1 & 401946 & 6551 & 959.639 & 10340 & 145.077 \\
\hline 300 & 500 & 2 & 1 & 0.01 & 0.1 & 1 & 4 & 0.1 & 0.3 & 0.1 & 0.5 & 1 & 0.5 & 0.01 & 0.3 & 0.7 & 0.03 & 0.001 & 0.01 & 1 & 401946 & 6551 & 959.639 & 10250 & 107.648 \\
\hline 300 & 500 & 2 & 1 & 0.01 & 0.1 & 1 & 4 & 0.1 & 0.3 & 0.1 & 0.5 & 1 & 0.5 & 0.01 & 0.3 & 0.1 & 0.05 & 0.001 & 0.01 & 1 & 401946 & 6551 & 959.639 & 10370 & 302.279 \\
\hline 300 & 500 & 2 & 1 & 0.01 & 0.1 & 1 & 4 & 0.1 & 0.3 & 0.1 & 0.5 & 1 & 0.5 & 0.01 & 03 & 0.1 & 0.07 & 0.001 & 0.01 & 1 & 401.945 & 6551 & 959.639 & 10550 & 272.3356 \\
\hline 300 & 500 & 2 & 1 & 0.01 & 0.1 & 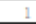 & 4 & 0.1 & 0.3 & 0.1 & 0.5 & 1 & 0.5 & 0.01 & 03 & 0.1 & 0.09 & 0.001 & 0.01 & 1 & 401946 & 6551 & 959.639 & 10550 & 242.392 \\
\hline 300 & 500 & 2 & 1 & 0.01 & 0.1 & 1 & 4 & 0.1 & 0.3 & 0.1 & 05 & 1 & 0.5 & 0.01 & 03 & 0.1 & 0.11 & 0.001 & 0.01 & 1 & 401946 & 6551 & 959.639 & 10500 & 212.449 \\
\hline 300 & 300 & 2 & 1 & 0.01 & 0.1 & 1 & 4 & 0.1 & 0.3 & 0.1 & 0.5 & 1 & 05 & 0.01 & 0.3 & 0.1 & 0.13 & 0.001 & 0.01 & 1 & 401946 & 6551 & 959.639 & 10430 & 182.506 \\
\hline 300 & 500 & 2 & 1 & 0.01 & 0.1 & 1 & 4 & 0.1 & 0.3 & 0.1 & 0.5 & 1 & 0.5 & 0.01 & 0.3 & 0.1 & 0.03 & 0.004 & 0.01 & 1 & 401945 & 6551 & 959.639 & 10500 & 331.009 \\
\hline 300 & 500 & 2 & 1 & 0.01 & 0.1 & 1 & 4 & 0.1 & 0.3 & 0.1 & 0.5 & 1 & 0.5 & 0.01 & 03 & 0.1 & 0.03 & 0.005 & 0.01 & , & 401945 & 6551 & 959.639 & 10500 & 330.35 \\
\hline 300 & 500 & 2 & 1 & 0.01 & 0.1 & 1 & 4 & 0.1 & 0.3 & 0.1 & 05 & 1 & 05 & 0.01 & 03 & 0.1 & 0.03 & 0.005 & 0.01 & 1 & 401945 & 6551 & 9959.639 & 10790 & 329.602 \\
\hline 300 & 500 & 2 & 1 & 0.01 & 0.1 & 1 & 4 & 0.1 & 0.3 & 0.1 & 0.5 & 1 & 05 & 0.01 & 03 & 0.1 & 0.03 & 0.01 & 0.01 & 1 & 401946 & 6551 & 959.639 & 10790 & 325.853 \\
\hline 300 & 500 & 2 & 1 & 0.01 & 0.1 & 1 & 4 & 0.1 & 0.3 & 0.1 & 0.5 & 1 & 0.5 & 0.01 & 0.3 & 0.1 & 0.03 & 0.012 & 0.01 & 1 & 401946 & 6551 & 959.639 & 10790 & 328.105 \\
\hline 300 & 500 & 2 & 1 & 0.01 & 0.1 & 1 & 4 & 0.1 & 0.3 & 0.1 & 0.5 & 1 & 0.5 & 0.01 & 0.3 & 0.1 & 0.03 & 0.001 & 0.02 & 1 & 401946 & 6551 & 959.639 & 10760 & 317.25 \\
\hline 300 & 500 & 2 & 1 & 0.01 & 0.1 & 1 & 4 & 0.1 & 0.3 & 0.1 & 0.5 & 1 & 05 & 0.01 & 03 & 0.1 & 0.03 & 0.001 & 0.04 & -1 & 401.945 & 6551 & 959.639 & 10590 & 287.307 \\
\hline 300 & 500 & 2 & 1 & 0.01 & 0.1 & 1 & 4 & 0.1 & 0.3 & 0.1 & 0.5 & 1 & 0.5 & 0.01 & 03 & 0.1 & 0.03 & 0.001 & 0.05 & 1 & 401.946 & 6551 & 959.639 & 10520 & 257,364 \\
\hline 300 & 500 & 2 & 1 & 0.01 & 0.1 & 1 & 4 & 0.1 & 0.3 & 0.1 & 0.5 & 1 & 0.5 & 0.01 & 0.3 & 0.1 & 0.03 & 0.001 & 0.05 & 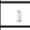 & 401946 & 6551 & 959.639 & 10540 & 227.421 \\
\hline 300 & 500 & 2 & 1 & 0.01 & 0.1 & 1 & 4 & 0.1 & 0.3 & 0.1 & 0.5 & 1 & 0.5 & 0.01 & 03 & 0.1 & 0.03 & 0.001 & 0.1 & 1 & 401946 & 6551 & 959.639 & 10470 & 197.478 \\
\hline 300 & 500 & 2 & 1 & 0.01 & 0.1 & 1 & 4 & 0.1 & 0.3 & 0.1 & 0.5 & 1 & 0.5 & 0.01 & 03 & 0.1 & 0.03 & 0.001 & 0.01 & 3 & 1623 & $1.10 \mathrm{~F}+06$ & $2.82 \mathrm{E}+04$ & $6.31 \mathbb{E}+05$ & $2.73 \mathrm{E}+05$ \\
\hline 300 & 500 & 2 & 1 & 0.01 & 0.1 & 1 & 4 & 0.1 & 0.3 & 0.1 & 0.5 & 1 & 0.5 & 0.01 & 0.3 & 0.1 & 0.03 & 0.001 & 0.01 & 4 & 3263 & $1.41 E+07$ & $122 \mathrm{E}+05$ & $1.05 \mathrm{E}+11$ & $7.11 \mathrm{~F}+06$ \\
\hline 300 & 300 & 2 & 1 & 0.01 & 0.1 & 1 & 4 & 0.1 & 0.3 & 0.1 & 0.5 & 1 & 0.5 & 0.01 & 0.3 & 0.1 & 0.03 & 0,001 & 0.01 & 5 & 6557 & $1.83 E+08$ & $5.08 E+05$ & $1.76 \mathrm{E}+13$ & $1.85 \mathrm{~F}+08$ \\
\hline 300 & 300 & 2 & 1 & 0.01 & 0.1 & 1 & 4 & 0.1 & 0.3 & 0.1 & 0.5 & 1 & 05 & 0.01 & 03 & 0.1 & 0.03 & 0.001 & 0.01 & 6 & 13180 & $2.36 \mathrm{E}+09$ & $2.19 E+06$ & $293 E+15$ & 4.79E +09 \\
\hline 300 & $-m$ & 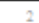 & 1 & 0.01 & 0.1 & & 4 & 0.1 & 03 & 0.1 & 05 & & 05 & 001 & 0.3 & 0.1 & 0.03 & 0.001 & 0.01 & 7 & 26490 & $3.04 \mathrm{TE}+10$ & $8.49 \mathrm{E}+05$ & $\mathrm{SSE}+17$ & $1.24 \mathrm{E}+11$ \\
\hline
\end{tabular}

